Submitted to Int. J. of Mod. Phys.

\title{
Spectral Anomaly and High Temperature Superconductors
}

\author{
Lan Yin and Sudip Chakravarty \\ Department of Physics and Astronomy \\ University of California Los Angeles \\ Los Angeles, California 90095-1547
}

(November 16, 1995)

\section{Contents}

\begin{tabular}{ll}
\hline I Introduction & 4 \\
\hline
\end{tabular}

$\begin{array}{llr}\text { II } & \text { Spectral anomaly } & 6\end{array}$

A The basic model . . . . . . . . . . . . . . . . . . . . 10

B Momentum distribution function . . . . . . . . . . . . . . . . . . . 12

C Matsubara Green's function . . . . . . . . . . . . . . . . . . . . . . . . . . 12

D Density of states . . . . . . . . . . . . . . . . . . . . . . 13

E Consistency of the basic model . . . . . . . . . . . . . . . . . . . . . . 14

F Vertex function . . . . . . . . . . . . . . . . . . . . . . 16

\begin{tabular}{llr}
\hline III & Perturbations & 17
\end{tabular}

A Interlayer tunneling in the normal state . . . . . . . . . . . . . . 18

1 Spectral function . . . . . . . . . . . . . . . . 20

B In-plane pairing interactions . . . . . . . . . . . . . . . . . . 21

1 Superconducting spectral function . . . . . . . . . . . . . 25

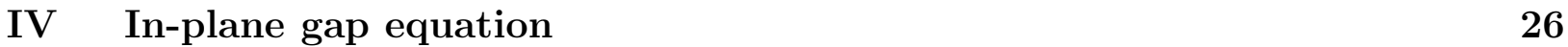

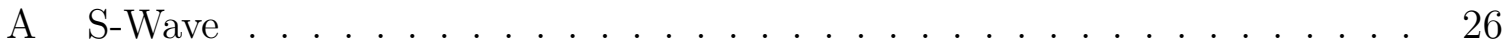


1 Density of states for $s$-wavd . . . . . . . . . . . . . . . . . 28

B D-Wave . . . . . . . . . . . . . . . . . . . . . . . 29

1 Density of states for $d$-wave . . . . . . . . . . . . . . . . . . . . 29

C Collective mode . . . . . . . . . . . . . . . . . . . . . . . 30

\begin{tabular}{|l|l|}
\hline V Josephson effect & 33 \\
\hline
\end{tabular}

\begin{tabular}{|lll}
\hline VI $\quad$ Interlayer pair tunneling & 36
\end{tabular}

\begin{tabular}{lll|} 
VII Spin-charge separation & 41 \\
\hline
\end{tabular}

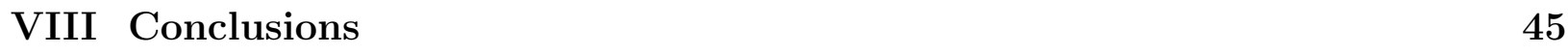

\begin{tabular}{ll}
\hline APPENDIXES & 47
\end{tabular}

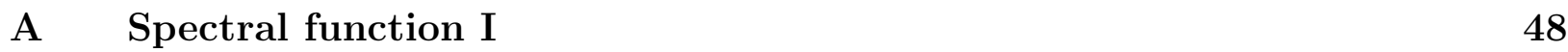

\begin{tabular}{lll}
\hline B $\quad$ Spectral function II & 49
\end{tabular} 


\begin{abstract}
Spectral anomaly for interacting Fermions is characterized by the spectral function $A\left(\left[k-k_{F}\right], \omega\right)$ satisfying the scaling relation $A\left(\Lambda^{y_{1}}\left[k-k_{F}\right], \Lambda^{y_{2}} \omega\right)=$ $\Lambda^{y_{A}} A\left(\left[k-k_{F}\right], \omega\right)$, where $y_{1}, y_{2}$, and $y_{A}$ are the exponents defining the universality class. For a Fermi liquid $y_{1}=1, y_{2}=1, y_{A}=-1$; all other values of the exponents are termed anomalous. In this paper, an example for which $y_{1}=1, y_{2}=1$, but $y_{A}=\alpha-1$ is considered in detail. Attractive interaction added to such a critical system leads to a novel superconducting state, which is explored and its relevance to high temperature cuprate superconductors is discussed.
\end{abstract}

Typeset using REVTEX 


\section{INTRODUCTION}

The normal state of high temperature superconductors is unusual: although the normal state has a metallic response and reflects gaplessness, its properties cannot be explained by Fermi liquid theory [1]. However, a microscopic theory of such a gapless non-Fermi liquid in dimensions $d \geq 2$ does not exist. In contrast, the breakdown of Fermi liquid theory in $d=1$ has been known since the pioneering work of Mattis and Lieb [4]. The absence of fermionic quasiparticles, spin-charge separation, and power-law correlation functions have been extensively discussed [5.6], and Haldane [7] has shown that these properties hold for a large class of interacting systems, the Luttinger liquids.

At the same time, a theory of high temperature superconductors has been proposed [8,9] in which Josephson tunneling of the Cooper pairs between the $\mathrm{CuO}$ layers plays an important role, and leads, in particular, to a striking dependence of the transition temperature on the number of such layers in the unit cell and to a high transition temperature by exploiting, in a very special way, the new energy scale proportional to the square of the tunneling matrix element divided by the in-plane band width. The validity of this theory depends on the nonFermi liquid nature of the normal state [10], as the special momentum conserving Josephson pair tunneling Hamiltonian cannot possibly be correct for a Fermi liquid. In view of the absence of a precise microscopic theory of the non-Fermi liquid, it is necessary to explore the extent to which a consistent theory can be developed based on general arguments because the consequences of this interlayer tunneling theory are quite tantalizing. It is our intention to present some general arguments in a reasonably complete form and to point out directions in which further improvements are essential.

In the present paper, we shall assume the existence of a higher dimensional non-Fermi liquid state and study the consequences for superconductivity. The essence of our arguments is a model of the one-particle Green's function and the corresponding spectral function. In constructing this model, we follow the stratagy of Galitskii and Migdal [13] who constructed a model for a Fermi liquid and examined its self consistency. The resulting theory is, of 
course, the same as the Landau theory of Fermi liquids. In the Galitski-Migdal model, the one-particle Green's function, analytically continued to the complex plane, contains simple poles on a second Riemann sheet, while the principal sheet is cut along the real frequency axis. The quasiparticle is a resonance in a many-body state, resembling the bare particle. In contrast, in our model, there are no poles but branch points on a second sheet, located infinitesimally close to the real axis. Thus, the non-Fermi liquid state is gapless and responds like a metal, but without quasiparticles. Such a model is motivated by the Luttinger liquid concept, but the spectral function is only qualitatively similar to that of the Luttinger liquid. In fact, in the present context, this model was briefly sketched by Wen [11]. The key element is the characterization of the non-Fermi liquid state as a critical state defined by a scale invariant spectral function [10]. This characterization is described by the term spectral anomaly, and, as in the theory of critical phenomena, the universality classes are distinguished by the critical exponents of the spectral function. Only a very special choice of the exponents leads to the fermi liquid model. In fact, we believe that all systems that respond as metals can be be described by our model. We also pay special attention to the Ward identity that relates the vertex function to the inverse Green's function. This is essential in building a consistent description of the non-Fermi liquid state.

The outline of our paper is as follows. In Sec. (II), we introduce the concept of spectral anomaly and examine the nature of the normal state. In Sec. (III), we consider the effect of various interactions added to the normal state described by spectral anomaly. In this section, we discuss only in-plane pairing interactions. In Sec. (IV), the superconducting gap equations with in-plane pairing interactions are solved for both $s$-wave and $d$-wave kernels, to examine the effect of the non-Fermi liquid behavior of the normal state. Sec. (V) contains a discussion of the Josephson effect and consititutes a qualitative justification of the interlayer pair-tunneling Hamiltonian discussed in Sec. (VI). It is worth noting that the analysis in this section bears some resemblance with a recent paper by Khlebnikov [12]. In Sec. (VI), we discuss the interlayer tunneling model, and, compute tunneling density of states with parameters as realistic as possible to compare with recent vacuum tunneling experiments. 
In Sec. (VII) we consider spin-charge separation and discuss how our basic model is changed in its presence. In the final concluding section, Sec. (VIII), we point out the directions in which our theory should be extended and improved.

\section{SPECTRAL ANOMALY}

For a finite system, the Källen-Lehmann representation [14] of the time-ordered oneparticle Green's function exhibits a discrete set of poles corresponding to the excitation frequencies of the system. In the infinite volume limit, the poles merge into a cut that runs along the real axis. Moreover, causality requires that the analytic continuation from the real axis to the complex plane be free of singularities in the first and the third quadrants. In fact, the only singularity on the principal sheet is a cut along the real axis.

To describe a Fermi liquid, Galitskii and Migdal [13] proposed a model of the one-particle Green's function, which contains singularities in the second and the fourth quadrants on a second sheet. These singularities were chosen by them to be simple poles, sufficiently close to the real axis to define long-lived quasiparticle states. The pole in the fourth quadrant corresponds to a quasiparticle and that in the second quadrant to a quasihole. (Throughout the paper we choose to work with a fixed chemical potential $\mu$ in a grand canonical ensemble rather than in a canonical ensemble for formal simplicity.). This model corresponds to the assumption that the matrix elements of the Fermion operators between the ground state and the exact eigenstates are sharply peaked at an energy that depends on the wavevector $\mathbf{k}$. The quasiparticle pole in the complex plane is the representation of the physical phenomena on the real frequency axis, as the theory of analytic continuation implies.

A simple pole corresponds to a unique energy-momentum dispersion of the quasiparticle: with a wave vector $\mathbf{k}$, we associate an energy $\varepsilon_{\mathbf{k}}$. And, with the renormalized wave function, the quasiparticles can be made to resemble the bare particles for energies asymptotically close to the Fermi energy. Other properties of the quasiparticles also follow from the assumptions of the model. The charge of the quasiparticle is unity and its spin $1 / 2$. 
The Galitskii-Migdal model can be summarized by the spectral function

$$
\begin{aligned}
A(k, \omega) & =-\frac{1}{\pi} \operatorname{Im} G_{\mathrm{R}}(k, \omega) \\
& =z_{k} \delta(\omega-(\varepsilon(k)-\mu)),
\end{aligned}
$$

where $G_{\mathrm{R}}$ is the retarded Green's function, and $z_{k}$ is the quasiparticle residue. As this model is valid for quasiparticles close to the Fermi surface, we can linearize the spectrum and write $\varepsilon(k)-\mu \approx v_{F}\left(k-k_{F}\right)$, where $k_{F}$ is the Fermi wave vector and $v_{F}$ is the Fermi velocity. Then, the spectral function has the scaling property

$$
A\left(\Lambda\left[k-k_{F}\right], \Lambda \omega\right)=\Lambda^{-1} A\left(\left[k-k_{F}\right], \omega\right),
$$

reflecting the gaplessness of the elementary excitations. The system is poised at criticality. That a normal Fermi system represents a critical system is, of course, understood. The important point, however, is that the criticality in a normal Fermi system does not usually lead to serious dynamical consequences. This is because the modes (the Fermionic excitations) decouple, as in the Gaussian model of classical critical phenomenon.

We would like to build a model in which the criticality of the Fermi system is more important. The simplest possible hypothesis is to assume that the analytic continuation of the Green's function to a second sheet contains branch points instead of simple poles. We postulate, therefore, a spectral function that satisfies the scaling relation

$$
A\left(\Lambda^{y_{1}}\left[k-k_{F}\right], \Lambda^{y_{2}} \omega\right)=\Lambda^{y_{A}} A\left(\left[k-k_{F}\right], \omega\right),
$$

where $y_{1}, y_{2}$, and $y_{A}$ are the exponents defining the universality class of the critical Fermi system. The values of the exponents other than the set $y_{1}=1, y_{2}=1$, and $y_{A}=-1$ will be termed anomalous, while this special set represents a Fermi liquid for which the branch points collapse into simple poles. The notion of a spectral function with anomalous exponents will be termed spectral anomaly.

The anomalous spectral function implies that a given $\mathbf{k}$ does not correspond to a single frequency but a continuum of frequencies. The creation operator of wave vector $\mathbf{k}$, applied 
to the ground state, creates a state that couples essentially to many eigenstates that cannot be expressed by the model of a weakly damped quasiparticle.

To establish continuity with the Fermi liquid model, as the exponents approach the Fermi liquid values, we make an important assumption. The location of the branch point is assumed to be in one-to-one correspondence with the states of the noninteracting system, which may be thought of as a remnant of Luttinger's theorem. This is by no means an obvious assumption, but holds for one-dimensional electron gas models [4, and also for gauge models [15] in higher dimensions.

From above, it is clear that we have not incorporated spin-charge separation in our definition, for we have assumed that the spectral function is singular at a single energy. The mathematical signature of spin-charge separation can be understood from the properties of a one-dimensional Luttinger liquid where the electron is a composite particle. The elementary particles are spinons that carry only spin and holons that carry only charge. As a result, the spectral function is singular at two energies. A generalization to include spin-charge separation is discussed in Sec. VII.

From the dispersion relation we can now determine the real part of the retarded Green's function, i.e,

$$
\operatorname{Re} G_{\mathrm{R}}\left(\left[k-k_{F}\right], \omega\right)=-\frac{\mathrm{P}}{\pi} \int_{-\infty}^{+\infty} d \omega^{\prime} \frac{\operatorname{Im} G_{\mathrm{R}}\left(\left[k-k_{F}\right], \omega\right)}{\omega-\omega^{\prime}} .
$$

Thus, the real part also satisfies the same scaling relation given by

$$
\operatorname{Re} G_{\mathrm{R}}\left(\Lambda^{y_{1}}\left[k-k_{F}\right], \Lambda^{y_{2}} \omega\right)=\Lambda^{y_{A}} \operatorname{Re} G_{\mathrm{R}}\left(\left[k-k_{F}\right], \omega\right) .
$$

From the generalized homogenity assumption, we also get

$$
A\left(\left[k-k_{F}\right], 0\right)=\frac{1}{\left|k-k_{F}\right|^{y_{A} / y_{1}}} A\left(\left[k-k_{F}\right] /\left|k-k_{F}\right|, 0\right)
$$

and

$$
A(0, \omega)=\frac{1}{|\omega|^{y_{A} / y_{2}}} A(0, \omega /|\omega|) .
$$


It is also possible to derive the momentum distibution function without any detailed knowledge of the function $A(k, \omega)$. From the Lehmann representation, the momentum distribution function $n(k)$ is given by

$$
n\left(k-k_{F}\right)=\int_{-\infty}^{0} d \omega A\left(\left[k-k_{F}\right], \omega\right)
$$

Using the scaling relation, we can write

$$
n\left(k-k_{F}\right)=\int_{-\infty}^{0} d \omega \Lambda^{-y_{A}} A\left(\Lambda^{y_{1}}\left[k-k_{F}\right], \Lambda^{y_{2}} \omega\right)
$$

Because $\Lambda$ is an arbitrary scale factor, we can chose $\Lambda^{y_{1}}\left|k-k_{F}\right|=1$. Then,

$$
n\left(k-k_{F}\right)=\left|k-k_{F}\right|^{\left(y_{2}+y_{A}\right) / y_{1}} \int_{-\infty}^{0} d x A\left(\operatorname{Sign}\left(k-k_{F}\right), x\right)
$$

The discontinuity present in a Fermi lquid is destroyed due to spectral anomaly. In a Fermi system, the occupation of a state, $n(k)$, cannot diverge, and the inequality $\left(y_{2}+y_{A}\right) / y_{1}>0$ must be satisfied. There is a superficial difficulty with the scaling argument above: the integral for $n(k)$ does not converge. Thus, the quantity $n(k)$ cannot be obtained from the scaling argument, and we need to restore the cutoff. However, we believe that the critical exponent is still given correctly by the scaling argument.

Consider now the time ordered Green's function, $G(k, \omega)$. It is

$$
G(k, \omega)=\operatorname{Re} G_{R}(k, \omega)-i \operatorname{Sign}(\omega) \operatorname{Im} G_{R}(k, \omega) .
$$

In terms of the time-ordered Greens function, we can write

$$
n(k)=\frac{-i}{2 \pi} \lim _{t \rightarrow 0^{+}} \int_{-\infty}^{+\infty} d \omega G(k, \omega) e^{i \omega t} .
$$

This integral can be broken up into three pieces. We write

$$
n(k)=\frac{-i}{2 \pi} \lim _{t \rightarrow 0^{+}}\left[\int_{-\infty}^{-\omega_{c}} d \omega G(k, \omega) e^{i \omega t}+\int_{-\omega_{c}}^{+\omega_{c}} d \omega G(k, \omega) e^{i \omega t}+\int_{+\omega_{c}}^{+\infty} d \omega G(k, \omega) e^{i \omega t}\right]
$$

The Green's function is assumed to be anomalous in the frequency range between $-\omega_{c}$ and $\omega_{c}$. In the second integral, we can set $t=0$ and apply the scaling form. The integral is well 
behaved becuse the singularity, as $\omega \rightarrow 0$, is softer than the Fermi liquid case $\left(\propto \frac{1}{\omega}\right)$. The first and the third integrals can be estimated by replacing $G$ by its correct asymptotic form,

which is $G(k, \omega) \rightarrow \frac{1}{\omega}$, as $\omega \rightarrow \infty$, resulting from the Fermion anticommutation relation. Finally, we find again that

$$
n(k)=\frac{1}{2}-C \operatorname{Sign}\left(k-k_{F}\right)\left|k-k_{F}\right|^{\left(y_{2}+y_{A}\right) / y_{1}},
$$

where $C$ is a constant.

\section{A. The basic model}

To proceed further, we introduce an explicit model that is defined by the retarded Green's function:

$$
G_{\mathrm{R}}(k, \omega)=\frac{g(\alpha) e^{i \phi}}{\omega_{c}^{\alpha}(\omega-\varepsilon(k)+i \delta)^{1-\alpha}},-\omega_{c} \leq \omega \leq \omega_{c}
$$

where $\phi$ is the phase, $\alpha$ is the anomaly exponent, and $\delta$ is an infinitesimal positive number. To conform to causality, the upper half plane should be free of singularities, and the cut is assumed to lie in the lower half plane. The quantity $g(\alpha)$ will be determined from the normalization. In a rough manner, one may include all nonsingular self energy effects in the definition of $\varepsilon(k)$. Therefore,

$$
-\frac{1}{\pi} \operatorname{Im} G_{\mathrm{R}}(k, \omega)=-g(\alpha) \frac{\sin \phi}{\pi} \frac{\theta(\omega-\varepsilon(k))}{\omega_{c}^{\alpha}(\omega-\varepsilon(k))^{1-\alpha}}-g(\alpha) \frac{\sin [\phi-\pi(1-\alpha)]}{\pi} \frac{\theta(\varepsilon(k)-\omega)}{\omega_{c}^{\alpha}(\varepsilon(k)-\omega)^{1-\alpha}} .
$$

Because the spectral function has to be positive, the phase $\phi$ must satisfy the inequality $0>\phi>-\pi \alpha$. This condition is not restrictive enough to fix $\phi$ uniquely. However, the time reversal symmetry is violated unless $\phi=-\alpha \pi / 2$. This is because of the invariance with respect to $\omega \rightarrow-\omega$, and $\varepsilon(k) \rightarrow-\varepsilon(k)$. Therefore,

$$
A(k, \omega)=g(\alpha) \frac{\sin \left(\frac{\pi \alpha}{2}\right)}{\pi \omega_{c}^{\alpha}} \frac{1}{|\varepsilon(k)-\omega|^{1-\alpha}}
$$


When $\varepsilon(k)=0$, our spectral function is similar to that of a Luttinger liquid without spincharge separation in $d=1$ [16, [1], but it is very different when $\varepsilon(k) \neq 0$. In a Luttinger liquid, the spectral function diverges with a power law as $\omega \rightarrow v_{F} k$ from above, vanishes for $-v_{F} k \leq \omega \leq v_{F} k$, and vanishes with a power law as $\omega \rightarrow-v_{F} k$ from below. In fact, the similarity with the one-dimensional system cannot be expected. The special kinematic constraints present in $d=1$ are missing in higher dimensions. Finally, note also the difference with the gauge models [15]: although the spectral functions are similar for $\varepsilon(k)=0$, for $k \neq 0$, the self energy in the gauge models is nonsingular.

To preserve the equal-time anticommutation relation of the Fermions, one must satisfy the sum rule $\int_{-\infty}^{\infty} d \omega A(k, \omega)=1$. If we integrate the spectral function, given in Eq. (2.17), we get instead:

$$
\int_{-\infty}^{\infty} d \omega A(k, \omega)=g(\alpha) \frac{\sin \left(\frac{\pi \alpha}{2}\right)}{\pi \alpha}\left[\left(1+\frac{\varepsilon(k)}{\omega_{c}}\right)^{\alpha}+\left(1-\frac{\varepsilon(k)}{\omega_{c}}\right)^{\alpha}\right] .
$$

If we choose

$$
g(\alpha)=\frac{\pi \alpha}{2 \sin \left(\frac{\pi \alpha}{2}\right)},
$$

the anticommutation relation will be satisfied if $|\varepsilon(k)| \ll \omega_{c}$, which is precisely the regime in which a scaling theory is appropriate.

We are still left with a minor problem with respect to the choice of the phase. For the exact retarded Green's function, the phase at $\omega=\infty, \phi(\infty)=0-\varepsilon$, because the imaginary part of $G_{\mathrm{R}}$ vanishes faster than the real part. As $\omega$ runs along the real axis, $\operatorname{Im} G_{\mathrm{R}}$ does not change sign and the phase remains in the lower half plane, reaching $\phi(-\infty)=\pi+\varepsilon$. For our Green's function, the phase remains in the lower half plane, varying between $-\alpha \pi / 2$ and $(\pi+\alpha \pi / 2)$. Clearly, the exact limits at $\pm \infty$ are violated, unless the scaling form is supplemented by the exact asymptotic limits of the Green's function. However, as long as $\alpha \neq 0$, discontinuities of the phase remain in our model; however, these disconitinuities appear to be physically inconsequential.

The time-ordered Green's function, $G(k, \omega)$, is given by 


$$
G(k, \omega)=g(\alpha) \omega_{c}^{\alpha}\left[\frac{e^{-i \frac{\pi \alpha}{2}} \theta(\omega)}{(\omega-\varepsilon(k)+i \delta)^{1-\alpha}}+\frac{e^{i \frac{\pi \alpha}{2}} \theta(-\omega)}{(\omega-\varepsilon(k)-i \delta)^{1-\alpha}}\right] .
$$

Note that the argument of the $\theta$-function is the frequency variable, $\omega$, and not $\varepsilon(k)$. For a Fermi liquid, there is no difference between these two cases; this is not so here. The correct $\arg u m e n t$ is always $\omega$ (the total energy), and not the kinetic energy $\varepsilon(k)$.

\section{B. Momentum distribution function}

The momentum distribution function is now given by

$$
n(k)=\frac{\alpha}{2 \omega_{c}^{\alpha}} \int_{-\omega_{c}}^{0} d \omega \frac{1}{|\varepsilon(k)-\omega|^{1-\alpha}} .
$$

We get

$$
n(k)=\frac{1}{2}\left[1-\operatorname{Sign}(\varepsilon(k))\left|\frac{\varepsilon(k)}{\omega_{c}}\right|^{\alpha}\right] .
$$

To understand what we meant previously by cutoff effects, let us attempt to derive $n(k)$ by scaling and extending the limit to $-\infty$. Then,

$$
n(k)=\frac{\alpha}{2}\left|\frac{\varepsilon(k)}{\omega_{c}}\right|^{\alpha} \int_{-\infty}^{0} d x \frac{1}{|x-\operatorname{Sign}(\varepsilon(k))|^{1-\alpha}} .
$$

We see that the singularity of $n(k)$ is captured correctly, but not the correct functional form. This is as it should be because the remaining integral does not converge. The calculation of $n(k)$ from the time-ordered Green's function gives, as before, the same result.

\section{Matsubara Green's function}

The Matsubara Green's function, $\mathcal{G}\left(k, \omega_{n}\right)$, can be obtained from the spectral function. Note that

$$
\mathcal{G}\left(k, \omega_{n}\right)=\int_{-\infty}^{\infty} d \omega^{\prime} \frac{A\left(k, \omega^{\prime}\right)}{i \omega_{n}-\omega^{\prime}}
$$

where $\omega_{n}=\pi(2 n+1) T$ are the Matsubara frequencies; $T$ is the temperature. Substituting the spectral function, we get 


$$
\mathcal{G}\left(k, \omega_{n}\right)=g(\alpha) \frac{\theta\left(\omega_{n}\right) e^{-i \frac{\pi \alpha}{2}}+\theta\left(-\omega_{n}\right) e^{i \frac{\pi \alpha}{2}}}{\omega_{c}^{\alpha}\left(i \omega_{n}-\varepsilon(k)\right)^{1-\alpha}},
$$

where we have used the integral

$$
\int_{0}^{\infty} d x \frac{x^{\alpha-1}}{(z+x)}=\frac{\pi}{\sin (\pi \alpha)} z^{\alpha-1}
$$

with $|\operatorname{Arg}(z)|<\pi$ and $1>\operatorname{Re} \alpha>0$.

\section{Density of states}

The density of states, $n(\omega)$, can be calculated from the spectral function. It is

$$
n(\omega)=\frac{\alpha}{2 \omega_{c}^{\alpha}} \int_{-\omega_{c}}^{\omega_{c}} d \omega^{\prime} \frac{\rho\left(\omega^{\prime}\right)}{\left|\omega^{\prime}-\omega\right|^{1-\alpha}}
$$

where

$$
\rho(\omega)=\sum_{k} \delta(\varepsilon(k)-\omega)
$$

If we choose $\rho(\omega) \approx \rho=$ const. for $-W \leq \omega \leq W$ and 0 otherwise, then

$$
n(\omega)=\frac{\rho}{2}\left[\left(\frac{W}{\omega_{c}}+\frac{\omega}{\omega_{c}}\right)^{\alpha}+\left(\frac{W}{\omega_{c}}-\frac{\omega}{\omega_{c}}\right)^{\alpha}\right] .
$$

We have also numerically calculated $n(\omega)$ for a more realistic $\rho(\omega)$. For small $\alpha$, the smearing is not enough to destroy the van Hove singularities, while for larger $\alpha$ it can completely smooth out the singularities.

In contrast to Luttinger liquids, the density of states does not vanish as $\omega \rightarrow 0$. In fact, it is essentially unchanged from the Fermi liquid value, obtained as $\alpha \rightarrow 0$. It would agree exactly with the Fermi liquid value if $\omega_{c}$ is chosen to be $W$. Once again, the correct

treatment of the cutoff cannot be overemphasized. The scaling trick, applied to the integral in Eq. (2.27) leads to

$$
n(\omega)=\frac{\rho \alpha}{2}\left|\frac{\omega}{\omega_{c}}\right|^{\alpha} \int_{-\infty}^{\infty} d x \frac{1}{|x-\operatorname{Sign}(\omega)|^{1-\alpha}},
$$


where the limits of the integral were set to $\pm \infty$. It would appear that the density of states vanishes as $\omega \rightarrow 0$. This is incorrect, because the remaining integral does not converge; the cutoffs cannot be set to $\pm \infty$. The vanishing of the density of states at the Fermi energy in a one-dimensional Luttinger liquid is a result of the special kinematic constraints that do not easily generalize to higher dimensions.

\section{E. Consistency of the basic model}

It is useful to consider Fermi liquid theory as an effective low energy theory of metals [8, 18 20]. We shall briefly repeat the arguments due to Polchinski [20. Imagine that we are considering an effective field theory below an energy scale $\omega_{c}$ at which there are strong Coulomb interactions. We want to find the effective low energy theory for energies smaller than $\omega_{c}$. The elementary excitations of the effective field theory underlying the GalitskiMigdal model are charged, spin-1/2, quasiparticles, similar to the original electrons.

Consider first the free action:

$$
\int d t d^{d} \mathbf{p}\left\{i \psi_{\mathbf{p} \sigma}^{*}(t) \partial_{t} \psi_{\mathbf{p} \sigma}(t)-(\varepsilon(\mathbf{p})-\mu) \psi_{\mathbf{p} \sigma}^{*}(t) \psi_{\mathbf{p} \sigma}(t)\right\}
$$

As we scale all energies by a factor $s<1$, the momenta must scale to the Fermi surface. To accomplish this scaling, we write

$$
\mathbf{p}=\mathbf{k}+\mathbf{l}
$$

where $\mathbf{k}$ is a vector on the Fermi surface and $\mathbf{l}$ is a vector orthogonal to it. Let

$$
\varepsilon(\mathbf{p})-\mu=l v_{F}(\mathbf{k})+O\left(l^{2}\right),
$$

where $v_{F}(\mathbf{k})$ is the Fermi velocity. As we scale the energies and the wave vectors, $E \rightarrow s E$, $\mathbf{k} \rightarrow \mathbf{k}$, and $\mathbf{l} \rightarrow s \mathbf{l}$, the action remains fixed, provided the dimension of the fermion operators is $s^{-1 / 2}$. Consider now all possible terms consistent with the symmetries and examine their

scaling. If there are no relevant operators, the effective low energy theory is self-consistent. 
(The generated mass term can be absorbed by redefining the fermi surface to be the true interacting Fermi surface)

The most important interaction is the four fermion operator:

$$
\begin{gathered}
\int d t d^{d-1} \mathbf{k}_{\mathbf{1}} d \mathbf{l}_{\mathbf{1}} d^{d-2} \mathbf{k}_{\mathbf{2}} d \mathbf{l}_{\mathbf{2}} d^{d-1} \mathbf{k}_{\mathbf{3}} d \mathbf{l}_{\mathbf{3}} d^{d-1} \mathbf{k}_{\mathbf{4}} d \mathbf{l}_{\mathbf{4}} V\left(\mathbf{k}_{1}, \mathbf{k}_{2}, \mathbf{k}_{3}, \mathbf{k}_{4}\right) \\
\psi_{\mathbf{p}_{1} \sigma}^{*}(t) \psi_{\mathbf{p}_{3} \sigma}(t) \psi_{\mathbf{p}_{2} \sigma^{\prime}}^{*}(t) \psi_{\mathbf{p}_{4} \sigma^{\prime}}(t) \delta^{d}\left(\mathbf{p}_{1}+\mathbf{p}_{2}-\mathbf{p}_{3}-\mathbf{p}_{4}\right)
\end{gathered}
$$

where we have assumed that the interaction is short-ranged. It can be seen that the interaction scales as $s$ times the dimension of the delta function. Let us assume, for the moment, that

$$
\begin{aligned}
\delta^{d}\left(\mathbf{p}_{1}+\mathbf{p}_{2}-\mathbf{p}_{3}-\mathbf{p}_{4}\right) & =\delta^{d}\left(\mathbf{k}_{1}+\mathbf{k}_{2}-\mathbf{k}_{3}-\mathbf{k}_{4}+\mathbf{l}_{1}+\mathbf{l}_{2}-\mathbf{l}_{3}-\mathbf{l}_{4}\right) \\
& \sim \delta^{d}\left(\mathbf{k}_{1}+\mathbf{k}_{2}-\mathbf{k}_{3}-\mathbf{k}_{4}\right)
\end{aligned}
$$

where we have ignored $\mathbf{l}$ in comparison to $\mathbf{k}$. This justified because the vectors $l$ scale to zero. Now the argument of the $\delta$-function does not depend on $s$, and the four fermion interaction scales as $s$, vanishing in the limit $s \rightarrow 0$. The interaction is irrelevant. The conclusion is almost correct except for the special kinematics implied by the Cooper channel scattering processes. For these processes, the delta function can be seen to be of order $s^{-1}$, transforming the dimension of the interaction to $s^{0}$. The net result is that the interaction is marginal [20]. Thus, except for special kinematics, the four fermion interaction is irrelevant. The argument, valid in dimensions $d \geq 2$, fails in $d=1$. In $d=1$, the $\delta$-function is always of order $s^{-1}$ and the interaction is always marginal.

We now turn to the consistency of our basic model. The action is

$$
\int d \omega d^{d} \mathbf{p} G^{-1}(\mathbf{p}, \omega) \psi_{\mathbf{p} \sigma}^{*}(\omega) \psi_{\mathbf{p} \sigma}(\omega)
$$

where the Green's function $G$ corresponds to that of the basic model. The dimension of $\psi_{\sigma}(\mathbf{p}, \omega)$ is $s^{-(3-\alpha) / 2}$ and that of $\psi_{\sigma}(\mathbf{p}, t)$ is $s^{-(1-\alpha) / 2}$. If we follow Polchinski, we see that the the four fermion interaction is irrelevant; even for those exceptional kinematics for which the interaction was marginal in the Fermi liquid case, it now scales as $s^{2 \alpha}$. The spectral anomaly 
is more stable than the Fermi liquid! In fact, in the weak coupling regime, it does not even allow a superconducting instability. In Sec. V, we shall see that this is indeed correct. The coupling has to reach a threshold before superconducting instability occurs. Remarkably enough, the Josephson pair tunneling Hamiltonian [9] describing interlayer tunneling is relevant and results in a superconducting instability for arbitrarily weak coupling. Such a term must therefore be included in the effective low energy theory.

\section{F. Vertex function}

In the normal state, the charge vertex and the current vertex satisfy the Ward identity 21

$$
\sum_{\mu} q_{\mu} \Gamma^{\mu}\left(k+\frac{q}{2}, k-\frac{q}{2}\right)=G^{-1}\left(k+\frac{q}{2}\right)-G^{-1}\left(k-\frac{q}{2}\right) .
$$

In this subsection, we shall use the notation $q \equiv\left(\mathbf{q}, q_{0} \equiv \omega\right)$ and the metric will be $(-1,1,1,1)$. Although this identity cannot fully determine the vertex, it is a strong constraint. In the limit $q \rightarrow 0$,

$$
G^{-1}\left(k+\frac{q}{2}\right)-G^{-1}\left(k-\frac{q}{2}\right) \approx \sum_{\mu} q_{\mu} \partial_{\mu} G^{-1}(k)+O\left(q^{3}\right)
$$

and the vertex can be chosen to be

$$
\Gamma^{\mu}\left(k+\frac{q}{2}, k-\frac{q}{2}\right)=\partial_{\mu} G^{-1}(k)
$$

In our model, the $T=0$ Green's function is given by

$$
G^{-1}(k)=g^{-1}(\alpha) \omega_{c}^{\alpha} e^{i \frac{\alpha \pi}{2} \operatorname{Sign}\left(k_{0}\right)}\left(k_{0}-\varepsilon_{\mathbf{k}}+i \delta k_{0}\right)^{1-\alpha}
$$

The vertex would be

$$
\begin{aligned}
\Gamma^{0}\left(k+\frac{q}{2}, k-\frac{q}{2}\right) & =\frac{(1-\alpha) e^{i \frac{\alpha \pi}{2} \operatorname{Sign}\left(k_{0}\right)} \omega_{c}^{\alpha}}{g(\alpha)\left(k_{0}-\varepsilon_{\mathbf{k}}+i \delta k_{0}\right)^{\alpha}}, \\
\Gamma^{i \neq 0}\left(k+\frac{q}{2}, k-\frac{q}{2}\right) & =\frac{(1-\alpha) e^{i \frac{\alpha \pi}{2} \operatorname{Sign}\left(k_{0}\right)} \omega_{c}^{\alpha}}{g(\alpha)\left(k_{0}-\varepsilon_{\mathbf{k}}+i \delta k_{0}\right)^{\alpha}} v^{i}(\mathbf{k})
\end{aligned}
$$


However, the sign of the imaginary part of $G^{-1}(k)$ changes when $k_{0}=0$. This leads to a $\delta$-fuction contribution to $\Gamma^{0}$ but has no influence on $\Gamma^{i \neq 0}$. So, we have

$$
\begin{aligned}
\lim _{q \rightarrow 0} \Gamma^{0}\left(k+\frac{q}{2}, k-\frac{q}{2}\right) & =\frac{\omega_{c}^{\alpha}}{g(\alpha)}\left[\frac{(1-\alpha) e^{i \frac{\alpha \pi}{2} \operatorname{Sign}\left(k_{0}\right)}}{\left(k_{0}-\varepsilon_{\mathbf{k}}+i \delta k_{0}\right)^{\alpha}}+2 i \sin \frac{\alpha \pi}{2}\left|\varepsilon_{\mathbf{k}}\right|^{1-\alpha} \delta\left(k_{0}\right)\right], \\
\lim _{q \rightarrow 0} \Gamma^{i \neq 0}\left(k+\frac{q}{2}, k-\frac{q}{2}\right) & =\frac{(1-\alpha) e^{i \frac{\alpha \pi}{2} \operatorname{Sign}\left(k_{0}\right)} \omega_{c}^{\alpha}}{g(\alpha)\left(k_{0}-\varepsilon_{\mathbf{k}}+i \delta k_{0}\right)^{\alpha}} v^{i}(\mathbf{k})
\end{aligned}
$$

Note that the vertex functions are singular and the system is always strongly interacting.

\section{PERTURBATIONS}

In the present section, we examine the effects of perturbations. The word perturbation is meant in a specific sense. We augment the basic model by adding various interactions which we call perturbations, but the solution of the resulting models is not perturbative. We consider two specific perturbations. The first is the interlayer tunneling in the normal state and the second is the in-plane BCS pairing interaction. The first perturbation is introduced to understand the incoherence of single particle tunneling. The second perturbation is designed to bring out certain features of the in-plane pairing.

It is convenient to use the language of functional integrals The amplitude in imaginary time, $Z$, is given by

$$
Z \sim \int \mathcal{D} \psi_{\sigma}^{*} \mathcal{D} \psi_{\sigma} e^{-S_{0}\left[\psi_{\sigma}^{*}, \psi_{\sigma}\right]-S_{I}\left[\psi_{\sigma}^{*}, \psi_{\sigma}\right]}
$$

where $S_{0}$ is

$$
S_{0}=-\frac{1}{\beta} \sum_{k, \sigma, \omega_{n}} \mathcal{G}^{-1}\left(k, \omega_{n}\right) \psi_{k \sigma}^{*}\left(\omega_{n}\right) \psi_{k \sigma}\left(\omega_{n}\right),
$$

where $\mathcal{G}\left(k, \omega_{n}\right)$ was derived earlier, and the spin index is denoted by $\sigma ; \beta$ is the inverse temperature. $\psi^{*}$ and $\psi$ are the Grassmann variables representing the fermions, and the Fourier series

$$
\psi_{k \sigma}(\tau)=\frac{1}{\beta} \sum_{\omega_{n}} e^{-i \omega_{n} \tau} \psi_{k \sigma}\left(\omega_{n}\right)
$$

relates the imaginary time Grassman variables to Grassman variables as a function of Matsubara frequencies. The various perturbations are contained in $S_{I}$. 


\section{A. Interlayer tunneling in the normal state}

Consider two identical layers, $i=1,2$, that are coupled by a tunneling matrix element, $t_{\perp}(k)$, assumed to be real. Then,

$$
S_{0}=-\frac{1}{\beta} \sum_{k, \sigma, \omega_{n}, i} \mathcal{G}^{-1}\left(k, \omega_{n}\right) \psi_{k \sigma}^{(i) *}\left(\omega_{n}\right) \psi_{k \sigma}^{(i)}\left(\omega_{n}\right) .
$$

The quantity $S_{I}$ is given by

$$
S_{I}\left[\psi_{\sigma}^{*}, \psi_{\sigma}\right]=\frac{1}{\beta} \sum_{k, \sigma, \omega_{n}} t_{\perp}(k)\left[\psi_{k \sigma}^{(1) *}\left(\omega_{n}\right) \psi_{k \sigma}^{(2)}\left(\omega_{n}\right)+(1 \rightarrow 2)\right] .
$$

Now, $S_{0}+S_{I}$ can be expressed in a $2 \times 2$ matrix form:

$$
S_{0}+S_{I}=-\frac{1}{\beta} \sum_{k, \sigma, \omega_{n}} \bar{\psi}_{k \sigma}^{*}\left(\omega_{n}\right) M\left(k, \omega_{n}\right) \bar{\psi}_{k \sigma}\left(\omega_{n}\right) .
$$

The column vector $\bar{\psi}_{k \sigma}\left(\omega_{n}\right)$ is

$$
\bar{\psi}_{k \sigma}\left(\omega_{n}\right)=\left(\begin{array}{c}
\psi_{k \sigma}^{(1)}\left(\omega_{n}\right) \\
\psi_{k \sigma}^{(2)}\left(\omega_{n}\right)
\end{array}\right)
$$

The matrix $M\left(k, \omega_{n}\right)$ is

$$
M\left(k, \omega_{n}\right)=\left(\begin{array}{cc}
\mathcal{G}^{-1}\left(k, \omega_{n}\right) & -t_{\perp}(k) \\
-t_{\perp}(k) & \mathcal{G}^{-1}\left(k, \omega_{n}\right)
\end{array}\right) .
$$

The excitation spectrum is given by the inverse of the determinant, $[\operatorname{det} M]^{-1}$, analyti-

cally continued to the real frequency axis. In particular, the singularities of the time-ordered Green's function are given by

$$
[\operatorname{det} M]^{-1}=\frac{\theta(\omega)}{e^{i \pi \alpha} \Omega_{+}(k, \omega)^{2(1-\alpha)}-t_{\perp}^{2}(k)}+\frac{\theta(-\omega)}{e^{-i \pi \alpha} \Omega_{-}(k, \omega)^{2(1-\alpha)}-t_{\perp}^{2}(k)},
$$

where

$$
\Omega_{ \pm}(k, \omega)=\left[g^{-1}(\alpha) \omega_{c}^{\alpha}\right]^{\frac{1}{(1-\alpha)}}(\omega-\varepsilon(k) \pm i \delta) .
$$

There are branch points in the second and the fourth quadrants of the complex plane at $\omega=\varepsilon(k) \pm i \delta$. The cuts are shown in Fig. (1). In addition to the cuts there are poles. Let us consider the case $\omega>0$. The poles are given by 


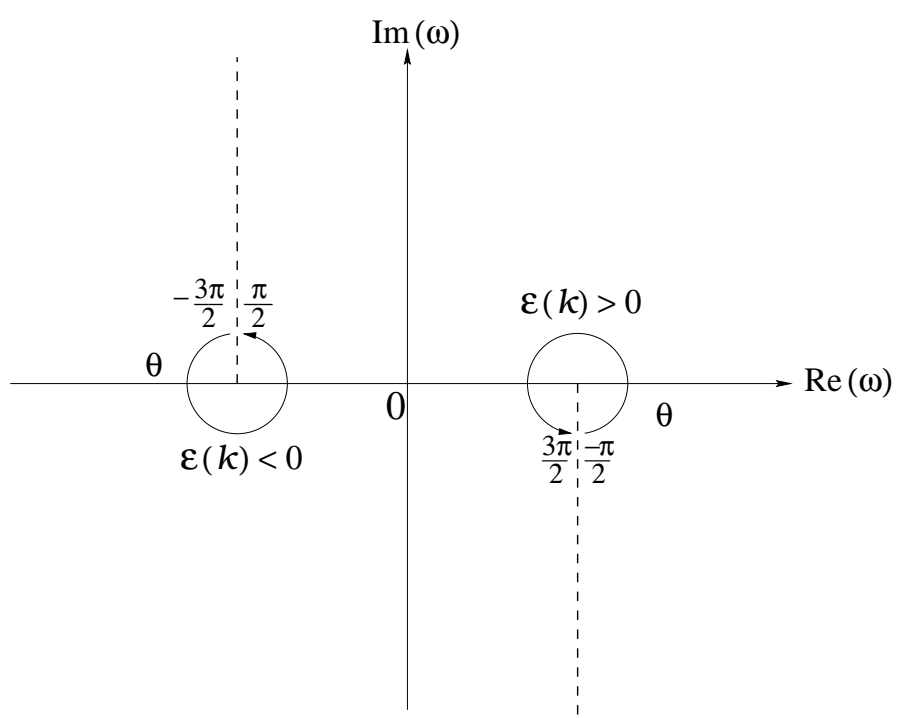

FIG. 1. Complex plane for the singularities of the Green's function for interlayer tunneling in the normal state.

$$
e^{i \pi \alpha} \Omega_{+}(k, \omega)^{2(1-\alpha)}=t_{\perp}^{2}(k) e^{2 i n \pi},
$$

where $n=0, \pm 1, \pm 2, \pm 3, \cdots$. If we define

$$
\omega-\varepsilon(k)+i \delta=|\omega-\varepsilon(k)| e^{i \theta},
$$

then the poles are the solutions of the following equations:

$$
\begin{gathered}
D^{-}(k, \omega)^{2(1-\alpha)}=t_{\perp}^{2}(k), \\
\theta=-\frac{\pi \alpha}{2(1-\alpha)}+\frac{n \pi}{(1-\alpha)},
\end{gathered}
$$

where

$$
D^{ \pm}(k, \omega)=\left[g^{-1}(\alpha) \omega_{c}^{\alpha}\right]^{\frac{1}{(1-\alpha)}}|\omega \pm \varepsilon(k)|
$$

To solve these equations, let us divide the complex plane into four regions (I)-(IV) as shown in Fig. (2): $0>\theta>-\frac{\pi}{2}$ (I), $\frac{\pi}{2}>\theta>0$ (II), $\pi>\theta>\frac{\pi}{2}$ (III), and $\frac{3 \pi}{2}>\theta>\pi($ IV). There are no solutions in regions (II) and (III), as it should be; otherwise, we would violate causality. The poles in regions (I) and (IV) are:

$$
\omega_{k}^{ \pm}=\varepsilon(k)+t_{\mathrm{eff}}(k)\left[ \pm \sin \frac{\pi / 2}{1-\alpha}+i \cos \frac{\pi / 2}{1-\alpha}\right]
$$




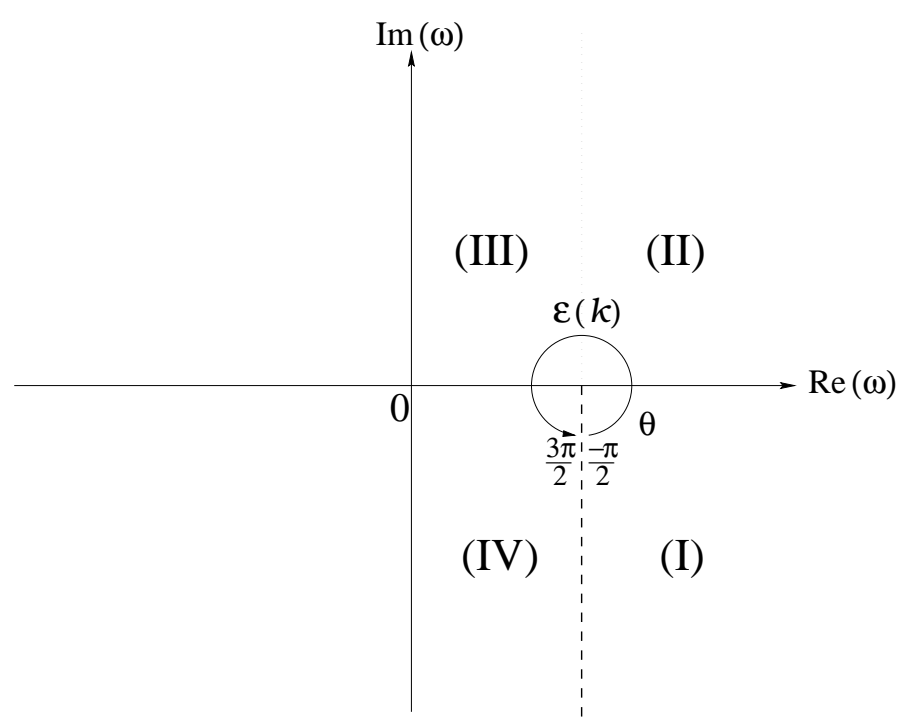

FIG. 2. Breakup into regions (I)-(IV) to solve for the poles of Green's function for interlayer tunneling in the normal state.

where

$$
t_{\mathrm{eff}}(k)=g(\alpha) t_{\perp}(k)\left(g(\alpha) \frac{t_{\perp}(k)}{\omega_{c}}\right)^{\frac{\alpha}{1-\alpha}}
$$

In the limit $\alpha \rightarrow 0$, these are the poles $\omega=\varepsilon(k) \pm t_{\perp}(k)$. As $\alpha$ increases, the poles spiral in and become degenerate at $\alpha=1 / 2$. Thus, at $\alpha=1 / 2$, no vestige of coherence is left [22]. For $\alpha>1 / 2$, the poles fall off the Riemann sheet. The poles for $\omega<0$ are analogous. Analytically continued to real frequencies, the time ordered Green's function is,

$$
\begin{gathered}
{[G(k, \omega)]^{-1}=\theta(-\omega)\left(\begin{array}{cc}
e^{-i \frac{\alpha \pi}{2}} \Omega_{-}(k, \omega)^{1-\alpha} & -t_{\perp}(k) \\
-t_{\perp}(k) & e^{-i \frac{\alpha \pi}{2}} \Omega_{-}(k, \omega)^{1-\alpha}
\end{array}\right)+} \\
\theta(\omega)\left(\begin{array}{cc}
e^{i \frac{\alpha \pi}{2}} \Omega_{+}(k, \omega)^{1-\alpha} & -t_{\perp}(k) \\
-t_{\perp}(k) & e^{i \frac{\alpha \pi}{2}} \Omega_{+}(k, \omega)^{1-\alpha}
\end{array}\right) .
\end{gathered}
$$

\section{Spectral function}

The spectral function is

$$
A_{i j}(k, \omega)=-\frac{1}{\pi} \operatorname{Sign}(\omega) \operatorname{Im}[G(k, \omega)]_{i j}=\frac{1}{t_{\perp}(k)} F_{i j}\left(\frac{\omega-\varepsilon(k)}{t_{\mathrm{eff}}(k)}\right),
$$


where $F_{i j}(x)$ is a dimensionless function. Its numerical evaluations are shown in Figs. (3) and (4); the explicit expressions are given in Appendix A. For the diagonal case, Fig. (3),

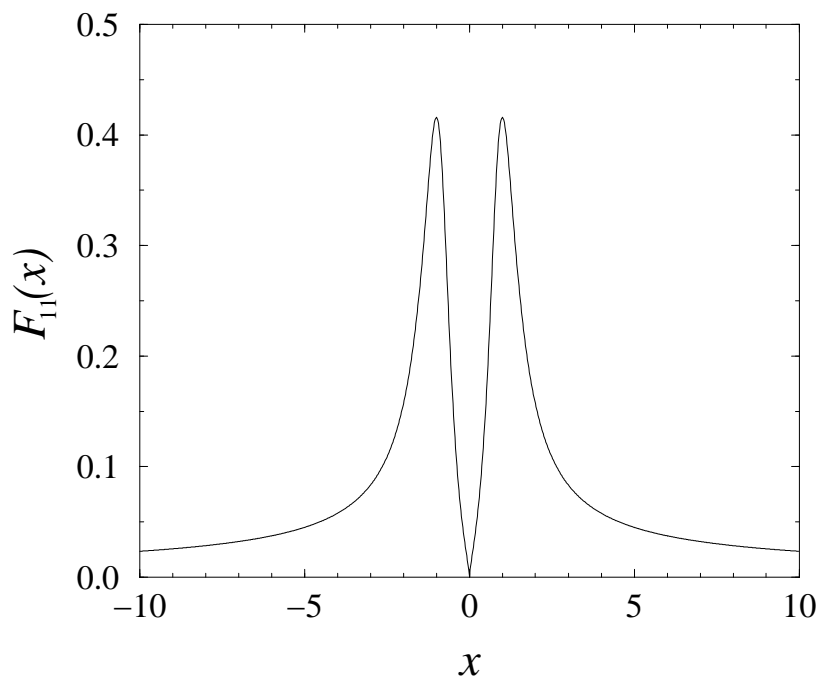

FIG. 3. Dimensionless diagonal spectral function for interlayer tunneling in the normal state; $(\alpha=0.25)$.

there are two symmetrically situated peaks, and the spectral weight vanishes at $\varepsilon(k)$. The shape of the peaks cannot be described by Lorentzians, because, at high frequencies, they are easily seen to fall off as the power law $\omega^{\alpha-1}$. Similarly, the off-diagonal spectral function is shown in Fig. (4). The, shape, once again, is distinctly non-Lorentzian, falling off as the power law $\omega^{2 \alpha-2}$. We have also calulated the corresponding density of states. For the diagonal part, it is almost constant over the entire region between $\omega_{c}$ and $-\omega_{c}$. For the off-diagonal part, it is essentially zero over the same range.

\section{B. In-plane pairing interactions}

Consider now a two-dimensional layer in which the electrons interact through an attractive pairing interaction. Later, we shall generalize to include bilayer systems. The interaction part, $S_{I}\left[\psi_{\sigma}^{*}, \psi_{\sigma}\right]$, is

$$
S_{I}=-\frac{1}{\beta^{3}} \sum_{\mathbf{k}, \mathbf{k}^{\prime}, \omega, \omega_{1}, \omega_{2}} V_{\mathbf{k}, \mathbf{k}^{\prime}} \psi_{\mathbf{k} \uparrow}^{*}\left(\omega_{1}\right) \psi_{-\mathbf{k} \downarrow}^{*}\left(\omega-\omega_{1}\right) \psi_{-\mathbf{k}^{\prime} \downarrow}\left(\omega-\omega_{2}\right) \psi_{\mathbf{k}^{\prime} \uparrow}\left(\omega_{2}\right)
$$




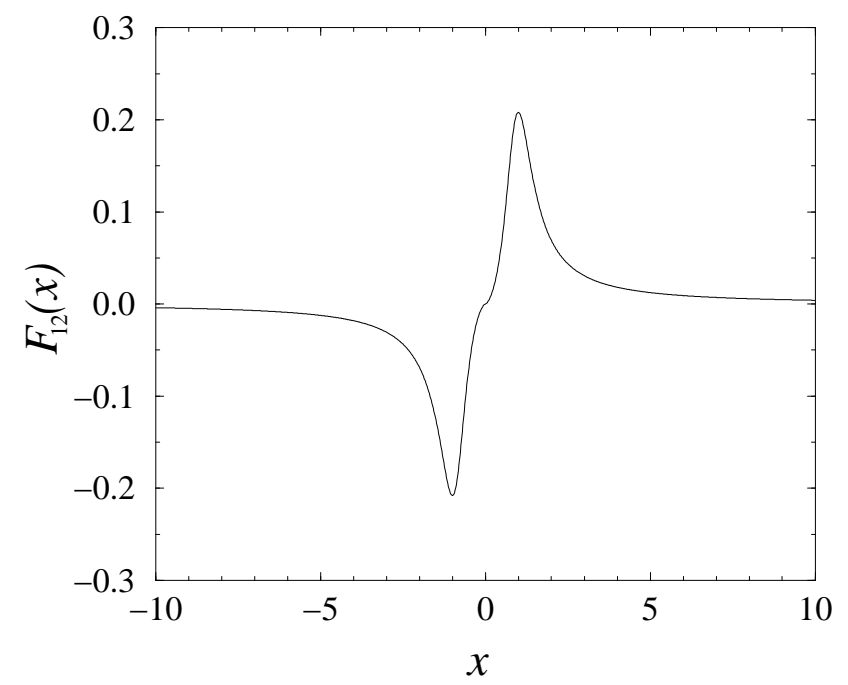

FIG. 4. Dimensionless off-diagonal spectral function for interlayer tunneling in the normal state. $(\alpha=0.25)$

For simplicity, the pairing interaction, $V_{k, k^{\prime}}$, will be assumed to be separable, i.e. ,

$$
V_{k, k^{\prime}}=\lambda_{k} \lambda_{k^{\prime}}
$$

Different pairing symmetries can be incoroprated by different choices of the functions $\lambda_{k}$. Introducing an auxiliary complex field, $c(\omega)$, and carrying out a Hubbard-Stratonovich transformation [23], we can write

$$
Z \sim \int \mathcal{D} c \mathcal{D} c^{*} \mathcal{D} \psi_{\sigma}^{*} \mathcal{D} \psi_{\sigma} e^{-\sum_{\omega} c^{*}(\omega) c(\omega)-S_{0}\left[\psi_{\sigma}^{*}, \psi_{\sigma}\right]-S_{I}\left[\psi_{\sigma}^{*}, \psi_{\sigma}\right]}
$$

The Grassmann variables can be integrated out by a linear shift, and we get

$$
Z \sim \int \mathcal{D} c \mathcal{D} c^{*} e^{-\sum_{\omega} c^{*}(\omega) c(\omega)+\operatorname{Tr} \ln A\left[c^{*}(\omega), c(\omega)\right]}
$$

where the matrix $A$ is

$$
A_{k_{1}, k_{2}}^{\omega_{1}, \omega_{2}}=\delta_{\mathbf{k}_{1}, \mathbf{k}_{2}}\left(\begin{array}{cc}
\mathcal{G}^{-1}\left(\mathbf{k}_{1}, \omega_{1}\right) \delta_{\omega_{1}, \omega_{2}} & \frac{\lambda_{k_{1}}}{\sqrt{\beta}} c\left(\omega_{1}-\omega_{2}\right) \\
\frac{\lambda_{k_{1}}}{\sqrt{\beta}} c^{*}\left(\omega_{2}-\omega_{1}\right) & -\mathcal{G}^{-1}\left(-\mathbf{k}_{1},-\omega_{1}\right) \delta_{\omega_{1}, \omega_{2}}
\end{array}\right)
$$

The saddle point corresponds to $c(\omega)=0, c^{*}(\omega)=0$, for $\omega \neq 0$. Then, denoting the superconducting gap, $\Delta_{k}$, by 


$$
\Delta_{k}=\frac{\lambda_{k}}{\sqrt{\beta}} c(0)
$$

we get

$$
\Delta_{k}=\lambda_{k} \sum_{k^{\prime}} \lambda_{k^{\prime}} \Delta_{k^{\prime}} \chi_{p}\left(k^{\prime}\right)
$$

where the pair susceptibility, $\chi_{p}(k)$, is

$$
\chi_{p}(k)=-\frac{1}{\beta} \sum_{\omega_{n}} \frac{1}{\mathcal{G}^{-1}\left(\mathbf{k}, \omega_{n}\right) \mathcal{G}^{-1}\left(-\mathbf{k},-\omega_{n}\right)+\Delta_{k}^{2}} .
$$

The excitation spectrum is determined by the singularities of the analytic continuation of $[\operatorname{det} A]^{-1}$, which is

$$
[\operatorname{det} A]^{-1}=-\frac{1}{\left(\varepsilon^{2}(k)-\omega^{2}-i \delta\right)^{1-\alpha} \omega_{c}^{2 \alpha} g^{-2}(\alpha)+\Delta_{k}^{2}} .
$$

In an earlier publication [10] the phase was chosen incorrectly; the mistake was pointed out to us by Abrahams [24]. Unfortunately, the same error appears to have propagated to later publications 25,26].

Clearly, there are branch points at $\omega= \pm \varepsilon(k)$. In addition, the spectrum consists of quasiparticle poles in the superconducting state. Consider $\varepsilon(k)>0$, and divide the complex plane into six regions as shown in Fig. (5). Let,

$$
(\omega-\varepsilon(k)+i \delta)^{1-\alpha}=|\omega-\varepsilon(k)|^{1-\alpha} e^{i(1-\alpha) \theta_{+}},-\frac{\pi}{2}<\theta_{+}<\frac{3 \pi}{2},
$$

and,

$$
(\omega+\varepsilon(k)-i \delta)^{1-\alpha}=|\omega+\varepsilon(k)|^{1-\alpha} e^{i(1-\alpha) \theta_{-}},-\frac{3 \pi}{2}<\theta_{-}<\frac{\pi}{2} .
$$

The quasiparticle poles are given by the solutions of the following equations:

$$
\left|\omega^{2}-\varepsilon^{2}(k)\right|^{1-\alpha} \omega_{c}^{2 \alpha} g^{-2}(\alpha)=\Delta_{k}^{2}
$$

and

$$
\theta_{+}+\theta_{-}=-\frac{\alpha \pi}{1-\alpha}+\frac{2 n \pi}{1-\alpha}
$$




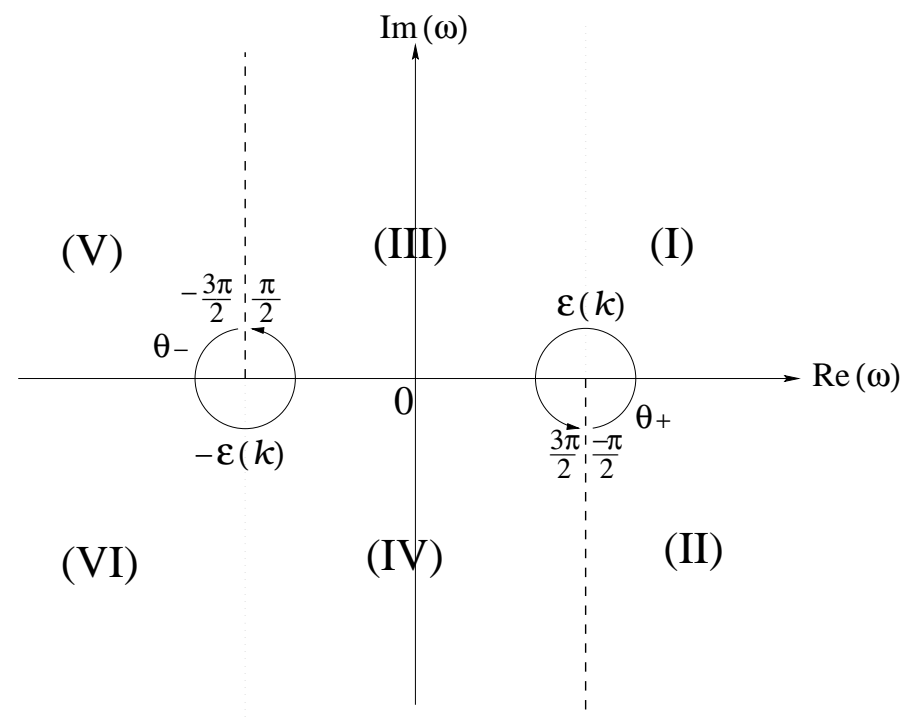

FIG. 5. Breakup of the complex plane into regions (I)-(VI) to obtain the poles of the superconducting Green's function.

where $n=0, \pm 1, \pm 2, \pm 3, \cdots$ There are solutions only in regions (II) and (V). These are given by

$$
\omega^{2}=\varepsilon^{2}(k)+\Delta_{\text {eff }}^{2}(k) e^{-i \frac{\alpha \pi}{1-\alpha}}
$$

where

$$
\Delta_{\text {eff }}(k)=g(\alpha) \Delta_{k}\left(g(\alpha) \frac{\Delta_{k}}{\omega_{c}}\right)^{\frac{\alpha}{1-\alpha}} .
$$

The quasiparticles are both damped and shifted in energy due to spectral anomaly. The effective gap $\Delta_{\text {eff }}(k)$ collapses at $\alpha=1$.

Analytically continued to real frequencies, the time ordered superconducting Green's function at $T=0$ is

$$
\begin{aligned}
{\left[G_{s}(\mathbf{k}, \omega)\right]^{-1}=} & \theta(-\omega)\left(\begin{array}{cc}
e^{-i \frac{\alpha \pi}{2}} \Omega_{-}(\mathbf{k}, \omega)^{1-\alpha} & \Delta_{\mathbf{k}} \\
\Delta_{\mathbf{k}} & -e^{i \frac{\alpha \pi}{2}} \Omega_{+}(-\mathbf{k},-\omega)^{1-\alpha}
\end{array}\right)+ \\
& \theta(\omega)\left(\begin{array}{cc}
e^{i \frac{\alpha \pi}{2}} \Omega_{+}(\mathbf{k}, \omega)^{1-\alpha} & \Delta_{\mathbf{k}} \\
\Delta_{\mathbf{k}} & -e^{-i \frac{\alpha \pi}{2}} \Omega_{-}(-\mathbf{k},-\omega)^{1-\alpha}
\end{array}\right)
\end{aligned}
$$




\section{Superconducting spectral function}

The superconducting spectral function is found to be

$$
A_{s}(k, \omega)=\frac{1}{\left|\Delta_{k}\right|} F_{s}\left(\frac{\omega}{\left|\Delta_{\text {eff }}(k)\right|}, \frac{\varepsilon(k)}{\left|\Delta_{\text {eff }}(k)\right|}\right),
$$

where $F_{s}(x, y)$ is dimensionless. The explicit expression is given in Appendix B. In Fig. (6) we show the dimensionless spectral function for $\varepsilon(k)>0$; for $\varepsilon(k)<0$, the figure should be symmetrically reflected. When $\varepsilon(k)=0$, the spectral function has two symmetrical peaks, in contrast to two delta functions at $\pm \Delta_{k}$, as in BCS theory. As the energy increases, the

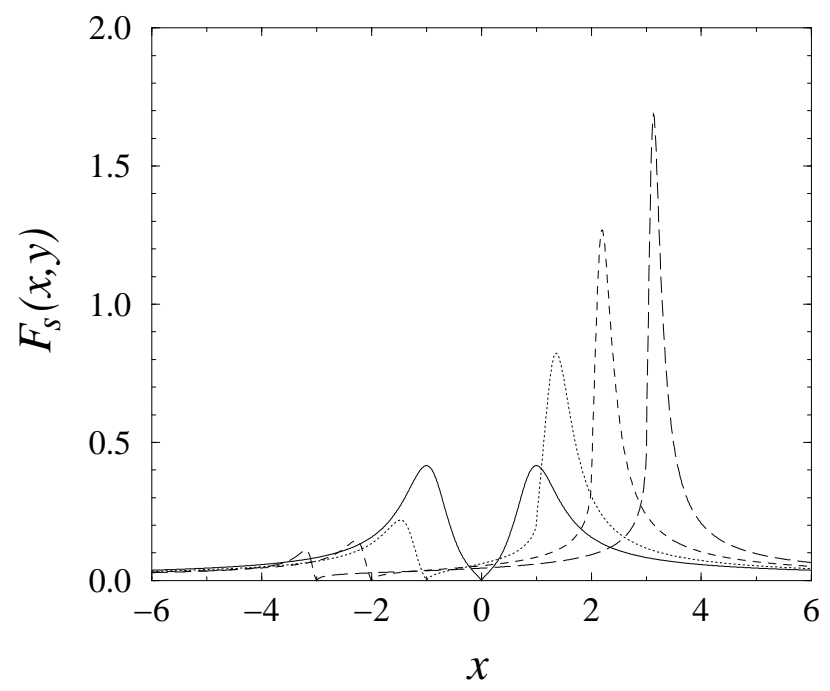

FIG. 6. Dimensionless superconducting spectral function. The solid, dotted, dashed, long-dashed lines are for $y=0,1,2,3 ;(\alpha=0.25)$.

weight of the peak on the positive frequency side grows and that on the negative frequency side decreases. The weight vanishes exactly at $-\varepsilon(k)$, but is finite everywhere else in contrast to BCS theory. The location of the peaks are the real parts of the quasiparticle energy. The energy dependence cannot be described by Lorentzians due to inherent power laws of the model. 


\section{IN-PLANE GAP EQUATION}

In this section we shall solve the gap equation that was derived in the previous section. We want to demonstrate that a critical value of the coupling is necessary if we assume only a in-plane pairing mechanism, and therefore by itself it is not such a promising mechanism for superconductivity if the normal state is a non-Fermi liquid. We also want to show that the density of states at zero frequency is finite even though the mean field theory leads to a pair amplitude. This is because the density of states is proportional to the momentum integral of the diagonal spectral function and the spectrum still contains gapless excitations arising from the cut in the Green's function. In solving the gap equations, it did not appear important to use realistic one-electron band structures and the approximation of a circular Fermi surface is used.

\section{A. S-Wave}

Consider a pairing interaction that is attractive around the Fermi surface over an energy range $\omega_{D}$ smaller than the cutoff $\omega_{c}$; for s-wave,

$$
V_{k, k^{\prime}}=-\lambda
$$

Then, the $T=0$ gap equation is

$$
\Delta_{0}=\frac{\rho \lambda}{2 \pi} \int_{-\infty}^{\infty} d \varepsilon \int_{-\omega_{D}}^{\omega_{D}} d y \frac{\Delta_{0}}{\left(y^{2}+\varepsilon^{2}\right)^{1-\alpha} \omega_{c}^{2 \alpha} g^{-2}(\alpha)+\Delta_{0}^{2}}
$$

For simplicity, we have chosen a cutoff prescription in which the integration over $\varepsilon$ is allowed to run between $\pm \infty$, but the remaining frequency integration is restricted between $\pm \omega_{D}$. Then, for $\alpha<1 / 2$, when $\Lambda \rightarrow \infty$, the gap $\Delta_{0}$ is found to be

$$
\Delta_{0}=\omega_{c} g^{-1}(\alpha)\left(\frac{\omega_{D}}{\omega_{c}}\right)^{1-\alpha}\left[\frac{1}{\pi} B\left(\frac{1}{2}, \frac{1}{2}-\alpha\right)\right]^{\frac{1-\alpha}{2 \alpha}}\left[\frac{(1-\alpha)}{\pi \alpha} \sin \left(\frac{\pi \alpha}{1-\alpha}\right)\left(1-\frac{\lambda_{c}}{\lambda}\right)\right]^{\frac{1-\alpha}{2 \alpha}}
$$

where 


$$
\rho \lambda_{c}=\frac{2 \alpha \pi g^{-2}(\alpha)}{B\left(\frac{1}{2}, \frac{1}{2}-\alpha\right)}\left(\frac{\omega_{c}}{\omega_{D}}\right)^{2 \alpha}
$$

is the critical value of the coupling necessary to obtain a non-zero superconducting gap, and $B(x, y)$ is the Eulerian beta function. Note that this critical value vanishes as $\alpha \rightarrow 0$, and one recovers the BCS result

$$
\lim _{\alpha \rightarrow 0} \Delta_{0}=2 \omega_{D} e^{-\frac{1}{\rho \lambda}}
$$

Consider now the equation for the transition temperature, $T_{c}$ :

$$
1=\frac{\lambda}{\beta_{c}} \sum_{\omega_{n}, k} \frac{1}{\left[\omega_{n}^{2}+\varepsilon^{2}(k)\right]^{1-\alpha} \omega_{c}^{2 \alpha} g^{-2}(\alpha)} .
$$

Once again, we choose to introduce cutoffs on the Matsubara frequencies at $\pm \omega_{D}$, but let the integration over $\varepsilon$ run over $\pm \infty$. Thus,

$$
1=\frac{\rho \lambda}{\beta_{c}} \int_{-\infty}^{\infty} d \varepsilon \sum_{n} \frac{1}{\omega_{c}^{2 \alpha} g^{-2}(\alpha)\left(\omega_{n}^{2}+\varepsilon^{2}\right)^{1-\alpha}} .
$$

We find that the that the transition temperature, $T_{c}$, is

$$
T_{c}=\frac{\omega_{D}}{\pi}\left(\frac{1}{2^{2 \alpha}-4 \alpha}\right)^{\frac{1}{2 \alpha}}\left(1-\frac{\lambda_{c}}{\lambda}\right)^{\frac{1}{2 \alpha}},
$$

where the critical value of $\lambda$ is given by

$$
\rho \lambda_{c}=\left(\frac{\omega_{c}}{\omega_{D}}\right)^{2 \alpha} \frac{2 \alpha \pi g^{-2}(\alpha)}{B\left(\frac{1}{2}, \frac{1}{2}-\alpha\right)} .
$$

The existence of the critical value of $\lambda$ was first pointed out by Balatsy [27], although his discussion of the reentrant phase transition appears to be incorrect due to an incorrect treatment of the cutoff. In the limit $\alpha \rightarrow 0, \lambda_{c} \rightarrow 0$, and

$$
T_{c}=\frac{e^{2} \omega_{D}}{2 \pi} e^{-\frac{1}{\rho \lambda}}
$$

The $4 \%$ discrepancy in the prefactor in comparison to the BCS is result is due to the different choice of the cutoff. This result disagrees with those obtained by Sudbø [25] and Muthukumar et al. 26]. Our calculation yields the BCS result in the limit $\alpha \rightarrow 0$. 


\section{Density of states for s-wave}

In Fig. (7) we show the superconducting density of states for various values of the parameters; see Appendix B for details. The surprising feature is the gaplessness, although a pseudogap persists. The square-root singularity of BCS is gone, but the peak is located at the effective gap. The low frequency behavior of the density of states $N(\omega)$ for $\alpha<1 / 2$

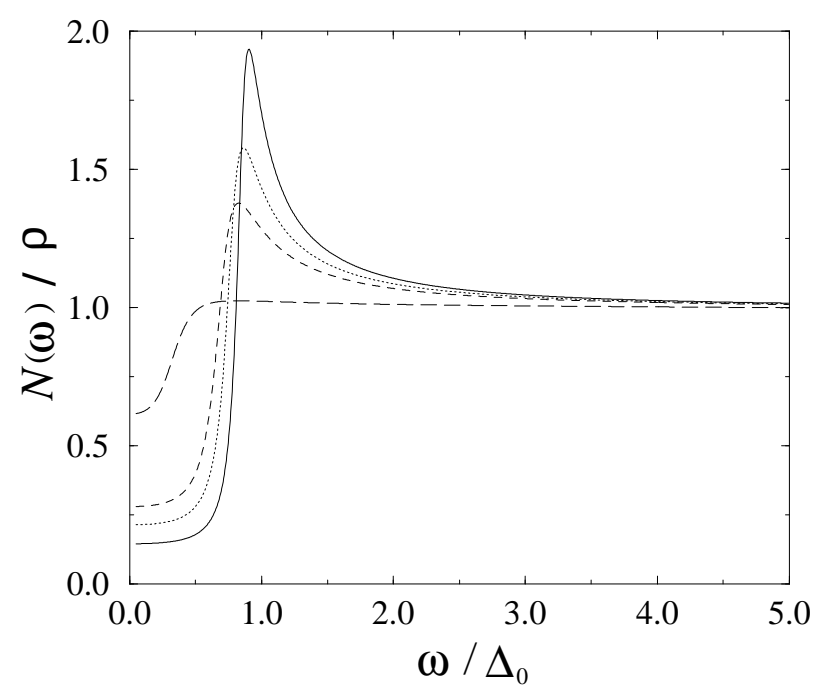

FIG. 7. Superconducting $s$-wave density of states for in-plane pairing. The solid, dotted, dashed, long-dashed curves are for $\alpha=0.05,0.075,0.01,0.25$.

is

$$
\begin{aligned}
\frac{N(\omega)-N(0)}{\rho}= & {\left[\frac{\alpha\left(2^{1-\alpha}-1\right)}{2-\alpha}\left(\frac{\omega_{c}}{g(\alpha) \Delta_{0}}\right)^{\alpha}\right]\left(\frac{\omega}{g(\alpha) \Delta_{0}}\right)^{2-\alpha} } \\
& +\left[\frac{\alpha}{2}\left(g(\alpha) \frac{\Delta_{0}}{\omega_{c}}\right)^{\frac{\alpha}{1-\alpha}} B\left(\frac{2-3 \alpha}{2(1-\alpha)}, \frac{2-\alpha}{2(1-\alpha)}\right)\right]\left(\frac{\omega}{g(\alpha) \Delta_{0}}\right)^{2},
\end{aligned}
$$

where

$$
\frac{N(0)}{\rho}=1-\frac{(\pi \alpha / 2(1-\alpha))}{\sin (\pi \alpha / 2(1-\alpha))}\left(g(\alpha) \frac{\Delta_{0}}{\omega_{c}}\right)^{\frac{\alpha}{1-\alpha}}
$$

The frequency dependence of the first term is nonanalytic and the coeffecient is essentially proportional to $\alpha$ for small $\alpha$; the cutoff and the gap dependences are weak. For, small $\alpha$, the quadratic term competes with the first term. The background, $N(0)$, vanishes in the 
limit $\alpha \rightarrow 0$, and one recovers the BCS result. The background also increases as $\alpha$ increases. Superconductivity is of course preserved because the saddle point of the action corresponds to broken gauge symmetry.

\section{B. D-Wave}

The zero temperature d-wave gap equation can be easily solved if we assume a separable kernel, where

$$
V_{k, k^{\prime}}=-\lambda \cos 2 \theta_{k} \cos 2 \theta_{k^{\prime}}
$$

and $\lambda>0$. Then the gap $\Delta_{k}$ is given by $\Delta_{k}=\Delta_{0} \cos 2 \theta_{k}$, and $\Delta_{0}$, in turn, is

$$
\Delta_{0}=\omega_{c} g^{-1}(\alpha)\left(\frac{\omega_{D}}{\omega_{c}}\right)^{1-\alpha}\left[\frac{(1-\alpha) B\left(\frac{1}{2}, \frac{1}{2}-\alpha\right)}{2 \alpha \pi B\left(\frac{1}{2}, \frac{3-\alpha}{2(1-\alpha)}\right)} \sin \left(\frac{\pi \alpha}{1-\alpha}\right)\left(1-\frac{\lambda_{c}}{\lambda}\right)\right]^{\frac{1-\alpha}{2 \alpha}}
$$

where

$$
\rho \lambda_{c}=4 \alpha\left(\frac{\omega_{c}}{\omega_{D}}\right)^{2 \alpha} \frac{\pi g^{-2}(\alpha)}{B\left(\frac{1}{2}, \frac{1}{2}-\alpha\right)} .
$$

The superconducting transition temperature can be found as before and it is

$$
T_{c}=\frac{\omega_{D}}{\pi}\left[\frac{1}{2^{2 \alpha}-4 \alpha}\left(1-\frac{\lambda_{c}}{\lambda}\right)\right]^{\frac{1}{2 \alpha}}
$$

The critical coupling, $\lambda_{c}$, is twice the s-wave critical temperature.

\section{Density of states for d-wave}

The superconducting density of states $N(\omega)$ is shown in Fig. (8). The logarithmic peak at the amplitude of the gap is smeared out and there are states at zero energy. The effect

of spectral anomaly increases as $\alpha$ increases. Consider now the low frequency behavior. For low frequencies, the integrals can be approximated and the result is

$$
\begin{aligned}
\frac{N(\omega)-N(0)}{\rho} & \approx\left[\frac{\alpha}{\sin \left(\frac{\alpha \pi}{2}\right)} B\left(\frac{3-\alpha}{2}, \frac{1+\alpha}{2}\right)-\frac{\alpha\left(1+2 \alpha-\alpha^{2}\right)}{2}\right]\left(\frac{\omega}{g(\alpha) \Delta_{0}}\right) \\
& \approx\left[1-\frac{3 \alpha}{2}\right]\left(\frac{\omega}{g(\alpha) \Delta_{0}}\right) .
\end{aligned}
$$




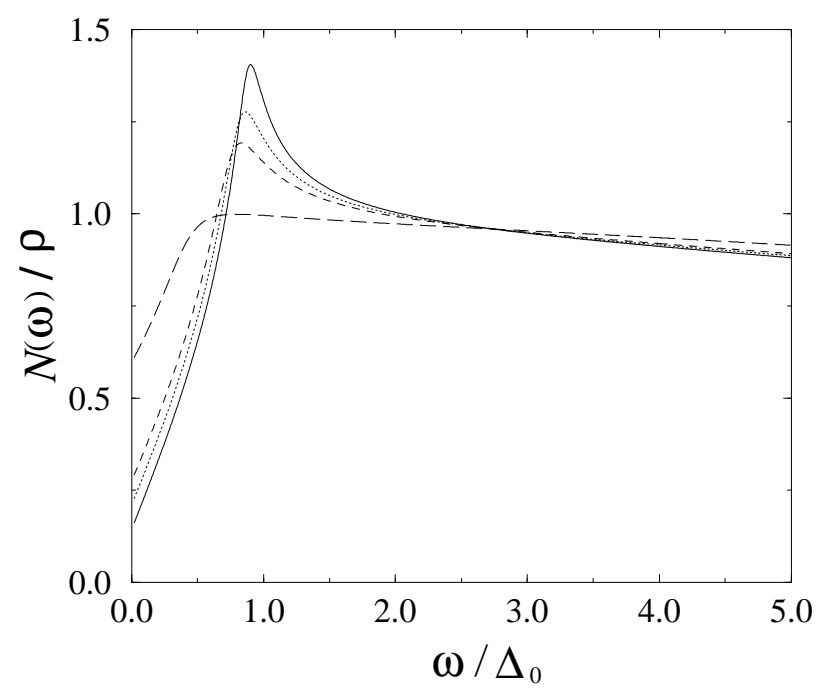

FIG. 8. Superconducting $d$-wave density of states for in-plane pairing. The solid, dotted, dashed, long-dashed curves are for $\alpha=0.05,0.075,0.01,0.25$.

Unlike s-wave, the low frequency exponent is unaffected by $\alpha$. The background term is

$$
N(0)=\rho\left[1-\frac{1}{2}\left(g(\alpha) \frac{\Delta_{0}}{\omega_{c}}\right)^{\frac{\alpha}{1-\alpha}} B\left(\frac{1}{2(1-\alpha)}, 1-\frac{\alpha}{2(1-\alpha)}\right)\right] .
$$

\section{Collective mode}

In the superconducting state, the pairing interaction not only gives rise to the gap, but also renormalizes the vertex and produces collective modes; possible long range interactions are important as well. In this section, we consider only the pairing interaction to examine the effect of non-fermi-liquid correlations.

In the random phase approximation and in the finite-temperature formalism, the vertex equation in the $T \rightarrow 0$ limit is given by 21

$$
\hat{\Gamma}_{s}(k, k+q)=\hat{\Gamma}_{n}(k, k+q)-\int \frac{d^{d+1} k^{\prime}}{(2 \pi)^{d+1}} V_{k, k^{\prime}} \tau_{3} \hat{\mathcal{G}}_{s}\left(k^{\prime}+q\right) \hat{\Gamma}_{s}\left(k^{\prime}, k^{\prime}+q\right) \hat{\mathcal{G}}_{s}\left(k^{\prime}\right) \tau_{3}
$$

We shall use the notation $q \equiv\left(\mathbf{q}, q_{0} \equiv \omega\right)$, where $q_{0}$ is now the Wick rotated frequency. The three Pauli matrices are the conventional $\tau_{1}, \tau_{2}$, and $\tau_{3}$. The equation is of course a

matrix equation. Near a collective mode, $\hat{\Gamma}_{s}(k, k+q)$ goes to $\infty$. The mode spectrum is then determined from the equation 


$$
\hat{\Gamma}_{s}(k, k+q)=-\int \frac{d^{d+1} k^{\prime}}{(2 \pi)^{d+1}} V_{k, k^{\prime}} \tau_{3} \hat{\mathcal{G}}_{s}\left(k^{\prime}+q\right) \hat{\Gamma}_{s}\left(k^{\prime}, k^{\prime}+q\right) \hat{\mathcal{G}}_{s}\left(k^{\prime}\right) \tau_{3}
$$

Consider S-wave pairing where $V_{k, k^{\prime}}=-\lambda<0$ and therefore $\hat{\Gamma}_{s}(q)=\hat{\Gamma}_{s}(k, k+q)$. Close to the phase mode, the vertex is a superposition of $\tau_{3}$ and $\tau_{2}$, but the coefficient of $\tau_{2}$ term diverges. Thus, the spectrum satisfies the following equation:

$$
\tau_{2} \approx \int \frac{d^{d+1} k^{\prime}}{(2 \pi)^{d+1}} \lambda \tau_{3} \hat{\mathcal{G}}_{s}\left(k^{\prime}+q\right) \tau_{2} \hat{\mathcal{G}}_{s}\left(k^{\prime}\right) \tau_{3}
$$

The right hand side of the above equation also contains a $\tau_{3}$ term, but it is negligible in deciding the spectrum. Substituting the expression for the Green's function

$$
\hat{\mathcal{G}}_{s}^{-1}(k)=\left(\begin{array}{cc}
\mathcal{G}^{-1}(k) & \Delta \\
\Delta & -\mathcal{G}^{-1}(-k)
\end{array}\right),
$$

we get

$$
1 \approx \lambda \int \frac{d^{d+1} k}{(2 \pi)^{d+1}} \frac{\mathcal{G}^{-1}(k+q) \mathcal{G}^{-1}(-k)+\Delta^{2}}{\left[|\mathcal{G}(k+q)|^{-2}+\Delta^{2}\right]\left[|\mathcal{G}(k)|^{-2}+\Delta^{2}\right]} .
$$

Subtracting the gap equation

$$
1=\lambda \int \frac{d^{d+1} k}{(2 \pi)^{d+1}} \frac{1}{|\mathcal{G}(k)|^{-2}+\Delta^{2}},
$$

we get

$$
0=\int \frac{d^{d+1} k}{(2 \pi)^{d+1}} \frac{\left[\mathcal{G}^{-1}\left(k+\frac{q}{2}\right)-\mathcal{G}^{-1}\left(k-\frac{q}{2}\right)\right]\left[\mathcal{G}^{-1}\left(-k-\frac{q}{2}\right)-\mathcal{G}^{-1}\left(-k+\frac{q}{2}\right)\right]}{\left[\left|\mathcal{G}\left(k+\frac{q}{2}\right)\right|^{-2}+\Delta^{2}\right]\left[\left[\left.\mathcal{G}\left(k-\frac{q}{2}\right)\right|^{-2}+\Delta^{2}\right]\right.} .
$$

In obtaining this equation, we have assumed particle-hole symmetry and have used the property $\mathcal{G}(k)=-\mathcal{G}\left(k^{\prime}\right)$ when $k_{0}=-k_{0}^{\prime}$ and $\varepsilon_{\mathbf{k}}=-\varepsilon_{\mathbf{k}^{\prime}}$.

In our model, the normal state Green's function is given by

$$
\mathcal{G}^{-1}(k)=g^{-1}(\alpha) \omega_{c}^{\alpha} e^{i \frac{\alpha \pi}{2} \operatorname{Sign}\left(k_{0}\right)}\left[i k_{0}-\varepsilon_{\mathbf{k}}\right]^{1-\alpha} .
$$

Note that the imaginary part of $\mathcal{G}^{-1}(k)$ changes sign at $k_{0}=0$. For small $q\left(q_{0}>0\right)$, we use the following approximations 


$$
\mathcal{G}^{-1}\left(k+\frac{q}{2}\right)-\mathcal{G}^{-1}\left(k-\frac{q}{2}\right) \approx\left\{\begin{array}{l}
2 i g(\alpha)^{-1} \sin \left(\frac{\alpha \pi}{2}\right) \omega_{c}^{\alpha}\left|\varepsilon_{\mathbf{k}}\right|^{1-\alpha} \text { for }\left|k_{0}\right|<\frac{q_{0}}{2} \\
\frac{(1-\alpha) e^{i \frac{\alpha \pi}{2}} \omega_{c}^{\alpha}}{g(\alpha)\left(i k_{0}-\varepsilon_{\mathbf{k}}\right)^{\alpha}}\left[i q_{0}-\mathbf{q} \cdot \mathbf{v}_{F}(\mathbf{k})\right] \text { for }\left|k_{0}\right|>\frac{q_{0}}{2}
\end{array}\right.
$$

and linearize the spectrum close to the Fermi surface. We get

$$
\begin{aligned}
0= & 4 q_{0} \sin ^{2}\left(\frac{\alpha \pi}{2}\right) \int \frac{|\epsilon|^{2-2 \alpha} d \epsilon}{\left[g^{-2}(\alpha) \omega_{c}^{2 \alpha}|\epsilon|^{2-2 \alpha}+\Delta^{2}\right]^{2}} \\
& +\left(q_{0}^{2}+\overline{\left|\mathbf{v}_{F} \cdot q\right|^{2}}\right)(1-\alpha)^{2} \int \frac{d \epsilon d \omega}{\left|\epsilon^{2}+\omega^{2}\right|^{\alpha}\left[g^{-2}(\alpha) \omega_{c}^{2 \alpha}\left|\epsilon^{2}+\omega^{2}\right|^{1-\alpha}+\Delta^{2}\right]^{2}}
\end{aligned}
$$

The limits of the integrals can be set to $\pm \infty$ with impunity. The notation $\bar{A}$ implies average over the Fermi surface. Finally, the spectrum for the phase mode is given by

$$
2 \alpha \sin \left(\frac{\alpha \pi}{2}\right) B\left(\frac{3-2 \alpha}{2-2 \alpha}, \frac{1-2 \alpha}{2-2 \alpha}\right)\left(\frac{g(\alpha) \Delta}{\omega_{c}}\right)^{\frac{\alpha}{1-\alpha}} q_{0} \Delta+(1-\alpha)^{2}\left(q_{0}^{2}+\overline{\left|v_{F} \cdot q\right|^{2}}\right)=0
$$

When $\alpha \ll 1$, to leading order in $\alpha$, after the analytic continuation $\Omega=i q_{0}$, this equation becomes

$$
-i \frac{\alpha^{2} \pi^{2}}{2} \Omega \Delta-\Omega^{2}+\overline{\left|\mathbf{v}_{F} \cdot \mathbf{q}\right|^{2}}=0
$$

There are two solutions

$$
4 \Omega_{1,2}=-i \alpha^{2} \pi^{2} \Delta \pm \sqrt{16 \overline{\left|\mathbf{v}_{F} \cdot \mathbf{q}\right|^{2}}-\alpha^{4} \pi^{4} \Delta^{2}}
$$

A propagating mode can exist only if

$$
q \xi_{0} \geq \frac{\pi^{2} \alpha^{2}}{4} \frac{1}{\sqrt{\left(\hat{v}_{F} \cdot \hat{q}\right)^{2}}}
$$

where $\xi_{0}=v_{F} / \Delta$ is the conventional definition of the coherence length. For a fixed $\alpha$, the mode is entirely overdamped as $q \rightarrow 0$. The damping of the collective phase mode is due to the coupling to the gapless single particle excitations of the non-Fermi liquid. As we have shown from calculations of the density of states, such gaplessness persists even in the superconducting state. In the presence of long range Coulomb interactions, charge fluctuations associated with the phase mode are energetically costly and the phase mode is irrelevant for superconductivity. 


\section{JOSEPHSON EFFECT}

It has been emphasized [10] that the Josephson pair-tunneling Hamiltonian (pairtunneling action in the language of the present paper) used in the interlayer tunneling of high temperature superconductors is not consistent with Fermi liquid theory, but requires a non-Fermi liquid Green's function as discussed in the present paper. Such a Hamiltonian can be presently understood only in the spirit of an effective field theory; similar arguments were also briefly described in Ref. 9]. In other words, we construct a low energy Hamiltonian based on certain general principles, such as symmetry. This approach is necessary because we do not, as yet, have a precise microscopic understanding of non-Fermi liquid theory. Thus, we are unable to explicitly integrate out the high energy degrees freedom to arrive at this Hamiltonian. The difficulty is also compounded by the fact that interesting results

arise only if, at the same time, we can suppress single particle tunneling between layers for which we can only give qualitative reasons. In particular, the incoherence of single particle tunneling between the layers, for sufficiently large $\alpha$, (See also the section on spin-charge separation, Sec. VIII.) is the clue that such a term should not be included in the effective low energy theory. This appears natural to us because an incoherent process is not a stationary process and its time evolution is not an oscillating exponential. The essential reason as to why single particle tunneling is incoherent is that the electronic quasipartcles are not well defined in a non-Fermi liquid, and the amplitude has to be reconstructed for an electron to tunnel between the layers. Such a process necessarily involves overlap of $N$-particle wave functions. In contrast, the superconducting wave function is an antisymmetrized wave function of Cooper pairs, and the tunneling matrix element only involves the overlap of a pair. It is the nature of a Bose condensed state that all pairs behave the same way. Thus, tunneling of Cooper pairs cannot be plagued with orthogonality catastrophe of $N$-particle wave functions. The aim of the present section is to give reasons as to why the pair tunneling Hamiltonian is reasonable from the point of view of effective field theory.

It is essential to realize that conventional Josephson effect in a Fermi liquid cannot be 
recast in the form of an effective Hamiltonian [10]. The well known expression for the critical current for two conventional superconductors with equal gaps [28],

$$
I_{c}=\frac{\pi \Delta(T)}{2 e R_{\mathrm{N}}} \tanh \left(\frac{\Delta(T)}{2 T}\right)
$$

where $R_{\mathrm{N}}$ is the normal state tunneling resistance, is unusual in two respects. As $T \rightarrow 0, I_{c}$ is a nonanalytic function of the gap $(\propto|\Delta|)$, and is analytic only close to $T_{c}$ (Note that, close to $T_{c}$, the magnitude of the Josephson effect, derived from Ginzburg-Landau theory [29], is proportional to $\Delta(\Delta / T)$ and the analytic result is due to the thermal smearing.). It is also remarkable that $I_{c}$ is independent of the cutoff, $\omega_{D}$, the Debye energy. The dependence on the Fermi energy is buried in the definition of $R_{\mathrm{N}}$. This is a manifestation of Fermi liquid theory. Consider the expression for the critical current at $T=0$, given by

$$
I_{c} \propto \sum_{\mathbf{k}, \mathbf{q}}\left|T_{\mathbf{k}, \mathbf{q}}\right|^{2} \frac{\left|\Delta_{\mathbf{k}}\right|}{E_{\mathbf{k}}} \frac{\left|\Delta_{\mathbf{q}}\right|}{E_{\mathbf{q}}} \frac{1}{E_{\mathbf{k}}+E_{\mathbf{q}}}
$$

where $E_{\mathbf{k}}=\sqrt{\varepsilon(\mathbf{k})^{2}+\left|\Delta_{\mathbf{k}}\right|^{2}}$. The integration over the momenta can be converted to energy integrations which extend only to the Debye energy. If the energy integrations can be extended to infinity with impunity, it can be seen by scaling the energy variables that $I_{c} \propto|\Delta|$; the correction is of order $\left(\frac{\Delta}{\omega_{D}}\right)$. This result hinges on the Fermi-liquid scaling of the single particle spectral function: $A(\Lambda k, \Lambda \omega)=\Lambda^{-1} A(k, \omega)$. Any non zero value of the scaling exponent, $\alpha$, will result in a cutoff dependence of the critical current $I_{c}$, and if this cutoff is much larger than the gap, the critical current can be an analytic function of the gap, proportional to $\Delta\left(\Delta / \omega_{c}\right)$. This allows us to reformulate Josephson effect in terms of an instantaneous pair tunneling hamiltonian on energy scales smaller than the cutoff.

In fact, explicit calculations [10] using the non-Fermi liquid spectral function of the present paper leads to

$$
I_{c} \propto\left\langle\left|T_{\mathbf{k}, \mathbf{q}}\right|^{2}\right\rangle v_{\mathrm{F}}^{-2} \Delta\left(\frac{\Delta}{\omega_{c}}\right) f\left(\alpha, \frac{\Delta}{\omega_{c}}\right)
$$

where the angular brackets imply an energy average and $f\left(\alpha, \frac{\Delta}{\omega_{c}}\right)$ is a complicated function. When $\frac{\Delta}{\omega_{c}} \ll 1, f\left(0, \Delta / \omega_{c}\right)=\omega_{c} / \Delta$, and we recover the Fermi liquid result. For $\alpha>1 / 4$, the 
dependence of $f\left(\alpha, \frac{\Delta}{\omega_{c}}\right)$ on $\frac{\Delta}{\omega_{c}}$ disappears entirely in the limit $\frac{\Delta}{\omega_{c}} \ll 1$, and it is a function of $\alpha$ alone. A closed form expression in the regime $\frac{1}{2}>\alpha>\frac{1}{4}$ was noted earlier [10]. For $\alpha$ not too small, but less than $\frac{1}{4}$, the dependence on $\frac{\Delta}{\omega_{c}}$ is weak.

We now construct a pair tunneling Hamiltonian. This Hamiltonian is certainly justified for $\alpha>\frac{1}{4}$, which is also the threshold at which incoherence sets in in the spin-chrage separated model (See Sec. VIII); for the case without spin-charge separation the threshold of incoherence is $\alpha=\frac{1}{2}$. In the tunneling between the layers, the transverse wave vector of an electron is conserved, and this conservation is an exact symmetry. Thus, consider the effective action

$$
S_{I}^{J}=\int d t^{\prime} d t d^{2} \mathbf{k}_{1} d^{2} \mathbf{k}_{2} \tilde{g}\left(\mathbf{k}_{1}, \mathbf{k}_{2},\left|t-t^{\prime}\right|\right)\left[\psi_{\mathbf{k}_{1} \sigma_{1}}^{*(1)}(t) \psi_{\mathbf{k}_{2} \sigma_{2}}^{*(1)}\left(t^{\prime}\right) \psi_{\mathbf{k}_{2} \sigma_{2}}^{(2)}(t) \psi_{\mathbf{k}_{1} \sigma_{1}}^{(2)}\left(t^{\prime}\right)+(1 \rightarrow 2)\right] .
$$

In view of the discussion above, we may set $\tilde{g}\left(\mathbf{k}_{1}, \mathbf{k}_{2},\left|t-t^{\prime}\right|\right)=\frac{g\left(\mathbf{k}_{1}, \mathbf{k}_{2}\right)}{\omega_{c}} \delta\left(t-t^{\prime}\right)$. Because in a non-Fermi lquid the tunneling process is so widely and uniformly spread over frequency that the Kernel is instantaneous. If we now repeat the scaling arguments of Sec. IIE, we see that the scaling dimension of this term at the non-Fermi liquid fixed point is $s^{-(1-2 \alpha)}$. This term is therefore relevant, diverging as $s \rightarrow 0$, as long as $\alpha<\frac{1}{2}$ and becomes marginal at $\alpha=\frac{1}{2}$. In contrast, we have seen in Sec. IIE that the conventional BCS pairing interaction (the in-plane mechanism in the interlayer tunneling theory), which involves integration over three independent wave vectors, is irrelevant, scaling as $s^{2 \alpha}$, hence vanishing as $s \rightarrow 0$. Indeed, we have seen in Sec. III that a coupling larger than a critical coupling is necessary to drive a superconducting transition, if we use a BCS pairing kernel.

It is reasonable to assume a separable form for $g\left(\mathbf{k}_{1}, \mathbf{k}_{2}\right)$ :

$$
g\left(\mathbf{k}_{1}, \mathbf{k}_{2}\right)=-t_{\perp}\left(\mathbf{k}_{1}\right) t_{\perp}\left(\mathbf{k}_{2}\right)
$$

where $t_{\perp}(\mathbf{k})$ is the single electron tunneling matrix element that can be determined from microscopic band structure calculations. An expression for $t_{\perp}(\mathbf{k})$ based on general considerations of symmetry and analyticity was proposed in Ref [9]; it is

$$
t_{\perp}(\mathbf{k})=\frac{t_{\perp}}{4}\left(\cos k_{x} a-\cos k_{y} a\right)^{2} .
$$


This expression for $t_{\perp}(\mathbf{k})$ is now confirmed from more detailed considerations of actual band structure computations 30 .

As in the original BCS theory, the pair tunneling action can be simplified, and a reduced action can be used for computations within the mean field approximation. This reduced Hamiltonian, which involves only pairing of states $(\mathbf{k} \uparrow)$ with $(-\mathbf{k} \downarrow)$, is

$$
-\int d t d^{2} \mathbf{k} \frac{t_{\perp}^{2}(\mathbf{k})}{\omega_{c}}\left[\psi_{\mathbf{k} \uparrow}^{*(1)}(t) \psi_{-\mathbf{k} \downarrow}^{*(1)}(t) \psi_{-\mathbf{k} \downarrow}^{(2)}(t) \psi_{\mathbf{k} \uparrow}^{(2)}\left(t^{\prime}\right)+(1 \rightarrow 2)\right]
$$

Note that it is misleading to consider the relevancy of this reduced action with the dimension

of the fermion operators determined from the fixed action of the normal state. The reduced action is equal to the original one only in the BCS subspace.

In addition to pair tunneling, we may also construct a particle-hole pair tunneling term given by

$$
S_{I}^{p-h}=\int d t^{\prime} d t d^{2} \mathbf{k}_{1} d^{2} \mathbf{k}_{2} \tilde{g}\left(\mathbf{k}_{1}, \mathbf{k}_{2},\left|t-t^{\prime}\right|\right)\left[\psi_{\mathbf{k}_{1} \sigma_{1}}^{*(1)}(t) \psi_{\mathbf{k}_{1} \sigma_{1}}^{(1)}\left(t^{\prime}\right) \psi_{\mathbf{k}_{2} \sigma_{2}}^{(2)}(t) \psi_{\mathbf{k}_{2} \sigma_{2}}^{*(2)}\left(t^{\prime}\right)+(1 \rightarrow 2)\right] .
$$

Similar to the single particle tunneling, the importance of this term is difficult to decide because this process is likely to be highly incoherent in a non-Fermi liquid for precisely the same reasons. In other words, the time dependence of the kernel may not be replaced by $\delta\left(t-t^{\prime}\right)$. If, on the other hand, the system has a tendency to form a spin density wave, this term may be more important than the pair tunneling term.

\section{INTERLAYER PAIR TUNNELING}

For interlayer tunneling in which there are both in-plane attractive interactions and Josephson pair hopping between the planes, we take $S_{0}$ to be

$$
S_{0}=-\frac{1}{\beta} \sum_{k, \sigma, \omega_{n}, i} \mathcal{G}^{-1}\left(\mathbf{k}, \omega_{n}\right) \psi_{\mathbf{k} \sigma}^{(i) *}\left(\omega_{n}\right) \psi_{\mathbf{k} \sigma}^{(i)}\left(\omega_{n}\right) .
$$

Similarly, 


$$
S_{I}^{\mathrm{i}-\mathrm{p}}=-\frac{1}{\beta^{3}} \sum_{\mathbf{k}, \mathbf{k}^{\prime}, \omega, \omega_{1}, \omega_{2}} V_{\mathbf{k}, \mathbf{k}^{\prime}}\left[\psi_{\mathbf{k} \uparrow}^{(1) *}\left(\omega_{1}\right) \psi_{-\mathbf{k} \downarrow}^{(1) *}\left(\omega-\omega_{1}\right) \psi_{-\mathbf{k}^{\prime} \downarrow}^{(1)}\left(\omega-\omega_{2}\right) \psi_{\mathbf{k}^{\prime} \uparrow}^{(1)}\left(\omega_{2}\right)+(1 \rightarrow 2)\right]
$$

and

$$
S_{I}^{\mathrm{J}}=-\frac{1}{\beta^{3}} \sum_{\mathbf{k}, \omega, \omega_{1}, \omega_{2}} T_{J}(\mathbf{k})\left[\psi_{\mathbf{k} \uparrow}^{(1) *}\left(\omega_{1}\right) \psi_{-\mathbf{k} \downarrow}^{(1) *}\left(\omega-\omega_{1}\right) \psi_{-\mathbf{k} \downarrow}^{(2)}\left(\omega-\omega_{2}\right) \psi_{\mathbf{k} \uparrow}^{(2)}\left(\omega_{2}\right)+(1 \rightarrow 2)\right]
$$

The term $S_{I}^{\mathrm{i}-\mathrm{p}}$ corresponds to a BCS in-plane pairing kernel as discussed in Secs. III and IV, while the term $S_{I}^{\mathrm{J}}$ is the interlayer pair tunneling term introduced in Sec. V. This model was discussed in some detail in Ref. [9], where, for simplicity, the single particle spectrum was assumed to be that of a Fermi liquid. The assumption was that for in-plane motions the quasiparticles are recovered in the superconducting state. This assumption is not entirely justified. As we have seen in the previous sections, the spectral function is in fact quite broad; see Fig. (6). Thus, in the present section we include properly the non-Fermi liquid features.

The Hubbard-Stratonovich transformation for the combined action is not entirely straightforward due to the local-in-k pair hopping term. First, let us rewrite the Josephson term as

$$
S_{I}^{\mathrm{J}}=2 \sum_{k, \omega}\left[\hat{A}(\mathbf{k}, \omega)^{*} \hat{A}(\mathbf{k}, \omega)-\hat{R}^{2}(\mathbf{k}, \omega)+\hat{I}^{2}(\mathbf{k}, \omega)\right]
$$

where we have ignored an unimportant single particle term that can be incorporated in the band structure. Here,

$$
\begin{aligned}
\hat{A}(\mathbf{k}, \omega)^{*} & =\frac{1}{2} \sqrt{\frac{T_{J}(\mathbf{k})}{\beta^{3}}} \sum_{\omega_{1}}\left[\psi_{\mathbf{k} \uparrow}^{(1) *}\left(\omega_{1}\right) \psi_{-\mathbf{k} \downarrow}^{(1) *}\left(\omega-\omega_{1}\right)+(1 \rightarrow 2)\right] \\
\hat{R}(\mathbf{k}, \omega) & =\frac{1}{4} \sqrt{\frac{T_{J}(\mathbf{k})}{\beta^{3}}} \sum_{\omega_{1}}\left[\psi_{\mathbf{k} \uparrow}^{(1) *}\left(\omega_{1}\right) \psi_{-\mathbf{k} \downarrow}^{(1) *}\left(\omega-\omega_{1}\right)+\psi_{-\mathbf{k} \downarrow}^{(1)}\left(\omega-\omega_{1}\right) \psi_{\mathbf{k} \uparrow}^{(1)}\left(\omega_{1}\right)-(1 \rightarrow 2)\right] \\
\hat{I}(\mathbf{k}, \omega) & =\frac{1}{4} \sqrt{\frac{T_{J}(\mathbf{k})}{\beta^{3}}} \sum_{\omega_{1}}\left[\psi_{\mathbf{k} \uparrow}^{(1) *}\left(\omega_{1}\right) \psi_{-\mathbf{k} \downarrow}^{(1) *}\left(\omega-\omega_{1}\right)-\psi_{-\mathbf{k} \downarrow}^{(1)}\left(\omega-\omega_{1}\right) \psi_{\mathbf{k} \uparrow}^{(1)}\left(\omega_{1}\right)-(1 \rightarrow 2)\right]
\end{aligned}
$$

The in-plane terms are decoupled, as usual, by using 


$$
\begin{aligned}
& \hat{B}_{1}^{*}(\omega)=\frac{1}{\beta^{3 / 2}} \sum_{k, \omega} \lambda(\mathbf{k}) \psi_{\mathbf{k} \uparrow}^{(1) *}(\omega) \psi_{-\mathbf{k} \downarrow}^{(1) *}\left(\omega-\omega_{1}\right) \\
& \hat{B}_{2}^{*}(\omega)=\frac{1}{\beta^{3 / 2}} \sum_{k, \omega} \lambda(\mathbf{k}) \psi_{\mathbf{k} \uparrow}^{(2) *}(\omega) \psi_{-\mathbf{k} \downarrow}^{(2) *}\left(\omega-\omega_{1}\right)
\end{aligned}
$$

We also include in the functional integral the factor

$$
\prod_{\omega} \exp \left[-B_{1}^{*}(\omega) B_{1}(\omega)-B_{2}^{*}(\omega) B_{2}(\omega)\right]
$$

and the factor

$$
\prod_{k, \omega} \exp \left[-2 A^{*}(\mathbf{k}, \omega) A(\mathbf{k}, \omega)-2 R^{2}(k, \omega)-2 I^{2}(k, \omega)\right]
$$

and integrate over $B_{1}(\omega), B_{1}^{*}(\omega), B_{2}(\omega), B_{2}^{*}(\omega), A(\mathbf{k}, \omega), A^{*}(\mathbf{k}, \omega)$, and over two real fields $R(\mathbf{k}, \omega)$, and $I(\mathbf{k}, \omega)$. A set of linear shifts

$$
\begin{aligned}
B_{1}(\omega) & \rightarrow B_{1}(\omega)-\hat{B}_{1}(\omega) \\
B_{2}(\omega) & \rightarrow B_{2}(\omega)-\hat{B}_{2}(\omega) \\
A_{k} & \rightarrow A_{k}-\hat{A}(\mathbf{k}, \omega) \\
R_{k} & \rightarrow R_{k}-i \hat{R}(\mathbf{k}, \omega) \\
I_{k} & \rightarrow I_{k}-\hat{I}(\mathbf{k}, \omega)
\end{aligned}
$$

eliminate all the quartic terms. The fermions can now be integrated out and the action can be written as

$$
\begin{aligned}
S=\sum_{\omega}\left[B_{1}^{*}(\omega) B_{1}(\omega)+B_{2}^{*}(\omega) B_{2}(\omega)\right] & +\sum_{k, \omega}\left[A^{*}(\mathbf{k}, \omega) A(\mathbf{k}, \omega)+R^{2}(\mathbf{k}, \omega)+I^{2}(\mathbf{k}, \omega)\right] \\
& -\ln \operatorname{Det}\left[M^{(1)} M^{(2)}\right]
\end{aligned}
$$

where $M^{(1)}$ and $M^{(2)}$ are two $2 \times 2$ matrices in $\mathbf{k}$ - $\omega$ space. The problem can be simplified if we note that at the saddle point

$$
\begin{aligned}
\lambda(\mathbf{k}) B_{1}(\omega)+ & \sqrt{T_{J}(\mathbf{k})}\left(A(\mathbf{k}, \omega)+\frac{i R(\mathbf{k}, \omega)}{2}+\frac{I(\mathbf{k}, \omega)}{2}\right)= \\
& {\left[\lambda(\mathbf{k}) B_{1}^{*}(\omega)+\sqrt{T_{J}(\mathbf{k})}\left(A^{*}(\mathbf{k}, \omega)+\frac{i R(\mathbf{k}, \omega)}{2}-\frac{I(\mathbf{k}, \omega)}{2}\right)\right]^{*} . }
\end{aligned}
$$


Therefore $R(\mathbf{k}, \omega)=I(\mathbf{k}, \omega)=0$. At the saddle point the determinant of the product of two matrices simplifies to

$$
\begin{aligned}
\operatorname{Det}\left[M^{(1)} M^{(2)}\right]=\frac{1}{\beta^{4}} & {\left[\mathcal{G}^{-1}(\mathbf{k}, \omega) \mathcal{G}^{-1}\left(-\mathbf{k}, \omega-\omega_{1}\right)+\beta^{-1}\left|\lambda(\mathbf{k}) B_{1}+\sqrt{T_{J}(\mathbf{k})} A(\mathbf{k})\right|^{2}\right] } \\
\times & {\left[\mathcal{G}^{-1}(\mathbf{k}, \omega) \mathcal{G}^{-1}\left(-\mathbf{k}, \omega-\omega_{1}\right)+\beta^{-1}\left|\lambda(\mathbf{k}) B_{2}+\sqrt{T_{J}(\mathbf{k})} A(\mathbf{k})\right|^{2}\right], }
\end{aligned}
$$

where all the fields are evaluated at $\omega=0: B_{1} \equiv B_{1}(0), B_{2} \equiv B_{2}(0)$, and $A(\mathbf{k}) \equiv A(\mathbf{k}, 0)$. On taking functional derivatives, we get,

$$
\begin{gathered}
0=\frac{\delta S}{\delta B_{1}^{*}}=B_{1}-\frac{1}{\beta} \sum_{k, \omega} \frac{\lambda(\mathbf{k})\left[\lambda(\mathbf{k}) B_{1}+\sqrt{T_{J}(\mathbf{k})} A(\mathbf{k})\right]}{\mathcal{G}^{-1}(\mathbf{k}, \omega) \mathcal{G}^{-1}(-\mathbf{k},-\omega)+\beta^{-1}\left|\lambda(\mathbf{k}) B_{1}+\sqrt{T_{J}(\mathbf{k})} A(\mathbf{k})\right|^{2}} \\
0=\frac{\delta S}{\delta A(k)^{*}}=2 A(k)-\frac{1}{\beta} \sum_{k, \omega} \frac{\sqrt{T_{J}(\mathbf{k})}\left[\lambda(\mathbf{k}) B_{1}+\sqrt{T_{J}(\mathbf{k})} A(\mathbf{k})\right]}{\mathcal{G}^{-1}(\mathbf{k}, \omega) \mathcal{G}^{-1}(-\mathbf{k},-\omega)+\beta^{-1}\left|\lambda(\mathbf{k}) B_{1}+\sqrt{T_{J}(\mathbf{k})} A(\mathbf{k})\right|^{2}} \\
0=\frac{\delta S}{\delta B_{2}^{*}}=B_{2}-\frac{1}{\beta} \sum_{k, \omega} \frac{\lambda(\mathbf{k})\left[\lambda(\mathbf{k}) B_{2}+\sqrt{T_{J}(\mathbf{k})} A(\mathbf{k})\right]}{\mathcal{G}^{-1}(\mathbf{k}, \omega) \mathcal{G}^{-1}(-\mathbf{k},-\omega)+\beta^{-1}\left|\lambda(\mathbf{k}) B_{1}+\sqrt{T_{J}(\mathbf{k})} A(\mathbf{k})\right|^{2}} .
\end{gathered}
$$

The saddle point is at $B_{1}=B_{2} \equiv B$. Now if we define the total gap $\Delta_{\mathbf{k}}$ by

$$
\Delta_{\mathbf{k}}=\lambda(\mathbf{k}) B+\sqrt{T_{J}(\mathbf{k})} A(\mathbf{k})
$$

then

$$
\Delta_{\mathbf{k}}\left[1-\chi(\mathbf{k}) T_{J}(\mathbf{k})\right]=\lambda(\mathbf{k}) \sum_{k^{\prime}} \lambda\left(\mathbf{k}^{\prime}\right) \Delta_{\mathbf{k}^{\prime}} \chi\left(\mathbf{k}^{\prime}\right)
$$

The pair susceptibility in terms of the total gap is the same as defined earlier. Note that the only difference from the result in Ref. [9] is the presence of non-Fermi liquid effects in the pair susceptibility. The derivation may appear to be a little more involved than necessary, but the formalism that we have set up could be quite useful if one is interested in the theory of collective modes [31]. This equation can be solved for any symmetry of the pairing interactions.

At $T=0$, this gap equation can be easily solved on the Fermi surface. Consider the anisotropic $s$-wave case [9]: $\lambda(\mathbf{k})=\sqrt{\lambda}$. At the Fermi surface

$$
\Delta_{\mathbf{k}_{F}}=\Delta_{0}+T_{J}\left(\mathbf{k}_{F}\right) \Delta_{\mathbf{k}_{F}} \chi\left(\mathbf{k}_{F}\right),
$$


where

$$
\Delta_{0}=\lambda \sum_{k^{\prime}} \Delta_{\mathbf{k}^{\prime}} \chi\left(\mathbf{k}^{\prime}\right)
$$

First consider the case $\alpha \ll 1 / 2$. Then, we can let the cutoff to $\infty$, because the integral converges. We get, if the Josephson pair hopping term dominates,

$$
\Delta_{\mathbf{k}_{F}} \approx \omega_{c} g^{-1}(\alpha)\left[\frac{T_{J}\left(\mathbf{k}_{F}\right)}{\omega_{c}}\right]^{\frac{1-\alpha}{1-2 \alpha}}\left[\frac{1}{2(1-\alpha) \sin \frac{\pi}{2(1-\alpha)}}\right]^{\frac{1-\alpha}{1-2 \alpha}} .
$$

This, as noted earlier [25], is encouraging, because local-in-k interlayer hopping produce a gap on the Fermi surface and there is no need for a critical coupling, necessary for purely in-plane mechanism discussed earlier. As $\alpha \rightarrow 1 / 2$, the above approximation of neglecting the cutoff $\omega_{D}$ cannot be used. However, the inegral can be easily approximated with the cutoff in place. The result, for $\alpha \rightarrow 1 / 2$, is

$$
\Delta_{\mathbf{k}_{F}} \approx \omega_{c} g^{-1}(\alpha)\left(\frac{\omega_{D}}{\omega_{c}}\right)^{1-\alpha} e^{-\frac{\pi \omega_{c}(1-\alpha)}{T_{J}\left(\mathbf{k}_{F}\right)}}
$$

Note that the precise results are not in agreement with those of Ref. [25]. This is due to an incorrect choice of the phase of the Green's function.

The density of states as $\omega \rightarrow 0$ can also be estimated, and surprisingly the behavior found earlier for the in-plane pairing is unchanged. We find for $\alpha \ll 1 / 2$ and $\omega \rightarrow 0$ :

$$
\frac{N(\omega)-N(0)}{\rho}=\left(\frac{\omega}{x}\right)^{2-2 \alpha}\left(\frac{2-2 \alpha}{2-\alpha}\right)
$$

where $\tilde{\Delta}_{0}<x<\tilde{\Delta}_{\max } ; \tilde{\Delta}_{\max }=\max \left[\Delta_{\text {eff }}(\mathbf{k})\right]$, and $\tilde{\Delta}_{0}=\min \left[\Delta_{\text {eff }}(\mathbf{k})\right]$. Thus, there is a finite density of states at $\omega=0$, and the low frequency behavior is $\omega^{2-\alpha}$.

It is interesting to compute tunneling density of states with a realistic Fermi surface corresponding to a two-layer high temperature superconductor, such as Bi2212 or YBCO, assuming an $s$-wave in-plane pairing kernel. For this we choose [32]

$$
\varepsilon_{k}=-0.5\left(\cos k_{x} a+\cos k_{y} a\right)+0.45 \cos k_{x} a \cos k_{y} a
$$

where $a$ is the lattice constant. The pair-hopping term $T_{J}(\mathbf{k})$ was chosen to be [9] 


$$
T_{J}(\mathbf{k})=\frac{T_{J}}{16}\left(\cos k_{x} a-\cos k_{y} a\right)^{4} .
$$

The other parameters are as follows: $T_{J}=0.05 \mathrm{eV}, \varepsilon_{F}=-0.315 \mathrm{eV}, \Delta_{0}=0.01 \mathrm{eV}$, and $\omega_{c}=0.15 \mathrm{eV}$. The results are shown in Fig. (9). The tunneling density of states are qualitatively similar to those of Renner and Fischer [33.

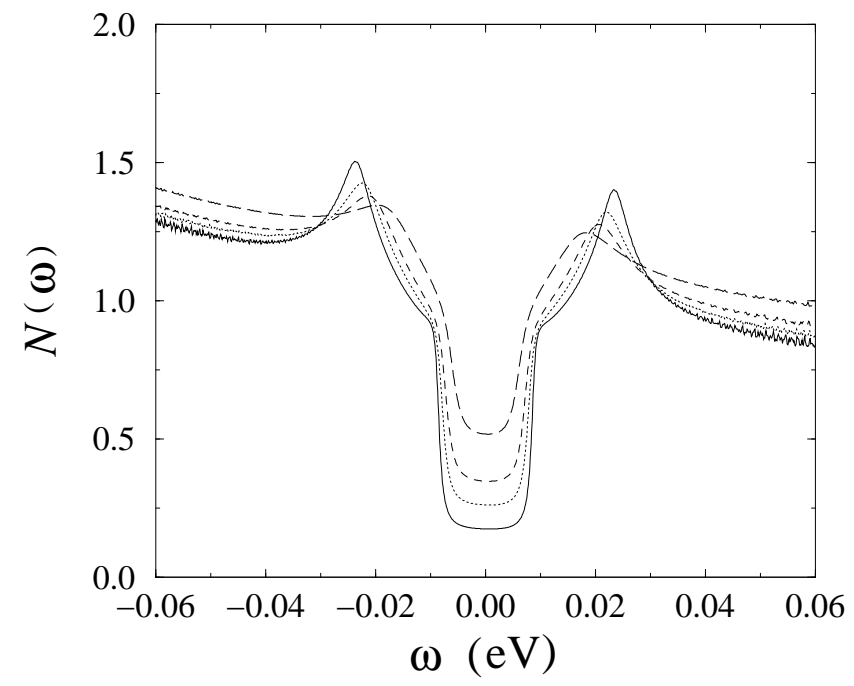

FIG. 9. Superconducting density of states for interlayer tunneling with realistic band parameters. The solid, dotted, dashed, long-dashed curves are for $\alpha=0.05,0.075,0.01,0.15$.

\section{SPIN-CHARGE SEPARATION}

In this section we shall consider a simple model for spin-charge separation [34]. The time-ordered Green's function at $T=0$ is

$$
G(k, \omega)=\frac{g(\alpha) e^{-i \frac{\pi \alpha}{2} \operatorname{Sign}(\omega)}}{\omega_{c}^{\alpha}\left(\omega-v_{s} k+i \delta \omega\right)^{\frac{1}{2}}\left(\omega-v_{\rho} k+i \delta \omega\right)^{\frac{1}{2}-\alpha}},
$$

where $v_{s}$ and $v_{\rho}$ are the spin and charge velocities $\left(v_{\rho}>v_{s}\right), k$ is measured from the Fermi surface, $g(\alpha)$ is the same as before, and $\omega_{c}$ is the cutoff. The corresponding spectral weight function is

$$
A(k, \omega)=\frac{1}{\pi}|\operatorname{Im} G(k, \omega)|
$$




$$
=\begin{aligned}
& \frac{1}{\pi}|G(k, \omega)| \sin \left(\frac{\pi \alpha}{2}\right), \text { when }\left(\omega-v_{\rho} k\right)\left(\omega-v_{s} k\right)>0 \\
& \frac{1}{\pi}|G(k, \omega)| \cos \left(\frac{\pi \alpha}{2}\right), \text { when }\left(\omega-v_{\rho} k\right)\left(\omega-v_{s} k\right)<0
\end{aligned}
$$

It has two power-law singularities and high frequency tails. The spectral weight in the region between the two singularities is larger by a factor of $\cot \left(\frac{\pi \alpha}{2}\right)$.

The momentum distribution function can be obtained by the formula $n(k)=$ $\int_{-\omega_{c}}^{0} A(k, \omega) d \omega$ and yields the value $1 / 2$ for $n(0)$. At finite $k$, and for $v_{\rho}|k|<<\omega_{c}$, we get

$$
n(k) \approx \frac{1}{2}-\frac{\operatorname{Sign}(k)}{2}\left[\left(\frac{1}{2}-\alpha\right) B\left(\frac{1}{2}, 1-\alpha\right)\left|\frac{\left(v_{\rho}-v_{s}\right) k}{\omega_{c}}\right|^{\alpha}-f\left(\alpha, \frac{v_{\rho}}{v_{s}}\right)\left|\frac{v_{s} k}{\omega_{c}}\right|^{\alpha}\right],
$$

where the function $f(\alpha, y)$ is given by the integral

$$
f(\alpha, y)=\alpha \int_{0}^{1} \frac{d x}{x^{\frac{1}{2}}(x+y-1)^{\frac{1}{2}-\alpha}} .
$$

We recover the earlier result for $n(k)$ without spin-charge separation when we set $v_{\rho}=v_{s}$, which results in $f(\alpha, 1)=1$. The density of states, $n(\omega)$, related to the spectral function by the formula $n(\omega)=\sum_{k} A(k, \omega)$, is constant when $v_{\rho}$ and $v_{s}$ are independent of $\omega$ and $k$.

If we consider, once again, the problem of tunneling between two planes, we find that the inverse of the matrix Green's function, $\hat{G}_{t}(k, \omega)$, is

$$
\left[\hat{G}_{t}(k, \omega)\right]^{-1}=\left(\begin{array}{cc}
G^{-1}(k, \omega) & -t_{\perp}(k) \\
-t_{\perp}(k) & G^{-1}(k, \omega)
\end{array}\right) .
$$

The quasiparticle poles are given by the equation $\operatorname{det}\left|G_{t}^{-1}(k, \omega)\right|=0$, more explicitly by

$$
\omega_{c}^{2 \alpha} g^{-2}(\alpha) e^{i \alpha \pi \operatorname{Sign}(\omega)}\left(\omega-v_{s} k+i \omega \delta\right)\left(\omega-v_{\rho} k+i \omega \delta\right)^{1-2 \alpha}=t_{\perp}^{2}(k)
$$

From the phase and the amplitude of this equation, we get

$$
\begin{aligned}
\operatorname{Sign}(\omega) \alpha \pi+\theta_{s}+\theta_{\rho}(1-2 \alpha) & =2 N \pi, \\
\omega_{c}^{2 \alpha}\left|\omega-v_{s} k\right|\left|\omega-v_{\rho} k\right|^{1-2 \alpha} & =g^{2}(\alpha) t_{\perp}^{2}(k) .
\end{aligned}
$$

For $k>0$ the equation for the phase restricts the poles in the shaded area shown in Fig(10). In general, there are two poles $\omega_{k}^{+}$and $\omega_{k}^{-}$, where $\operatorname{Re} \omega_{k}^{+}>v_{\rho} k, \operatorname{Im} \omega_{k}^{+}<0$, and $\operatorname{Re} \omega_{k}^{-}<v_{s} k$, 


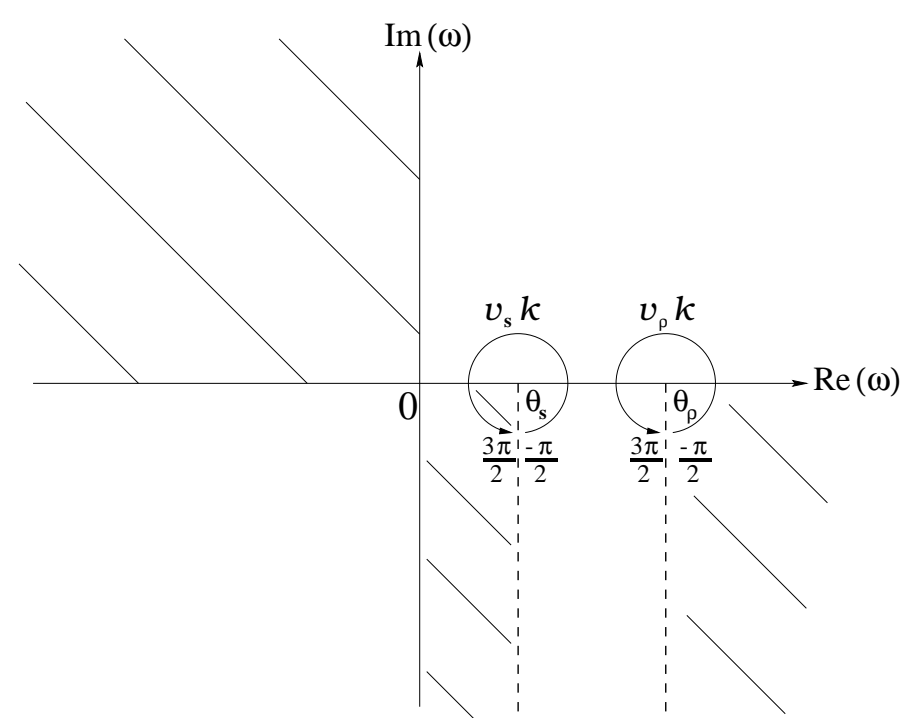

FIG. 10. Complex plane depiction of the singularities of the Green's function for the spin-charge separated model.

$\omega_{k}^{-} \operatorname{Im} \omega_{k}^{-}<0$. However, when $\alpha>\frac{1}{4}$ and $t_{\perp}(k)$ is less than a critical value, the pole $\omega_{k+}$ is absent, which clearly indicates supression of coherent tunneling. This is an important respect in which the spin-charge separated case differs from the previously discussed case of no spin-charge separation. To understand why the pole disappears, consider the equation for the phase:

$$
\alpha \pi+\theta_{s}+\theta_{\rho}(1-2 \alpha)=0
$$

Because $\theta_{\rho}>-\frac{\pi}{2}, \theta_{s}$ is bounded by $\theta_{s} \leq \frac{\pi}{2}(1-4 \alpha)$. When $\alpha>\frac{1}{4}$, this inequality confines the pole in the shaded area as shown in Fig(11). For $\omega$ belonging to the shaded area, the left hand side of the amplitude equation has the minimum: $\omega_{c}^{2 \alpha}\left(v_{\rho} k-\right.$ $\left.v_{s} k\right)^{2(1-\alpha)}|\sec (\phi(\alpha))||\tan (\phi(\alpha))|^{1-2 \alpha}$, where $\phi(\alpha)=\frac{\pi}{2}(1-4 \alpha)$. For $g^{2}(\alpha) t_{\perp}^{2}(k)$ less than this minimum value, the equation for the amplitude can not be satisfied. So the pole $\omega_{k+}$ does not exist for $\alpha>\frac{1}{4}$ and $t_{\perp}(k)$ less than a critical value given by the minimum.

We now discuss the superconducting properties. The calculations are similar to those discussed earlier. For S-wave pairing, the gap is

$$
\Delta=g^{-1}(\alpha) \omega_{D}\left[\frac{h\left(\alpha, \frac{v_{s}}{v_{\rho}}\right)}{h^{\prime}\left(\alpha, \frac{v_{s}}{v_{\rho}}\right) B\left(\frac{1}{1-\alpha}, \frac{1-2 \alpha}{1-\alpha}\right)}\right]^{\frac{1-\alpha}{2 \alpha}}\left(1-\frac{\lambda_{c}}{\lambda}\right)^{\frac{1-\alpha}{2 \alpha}}
$$




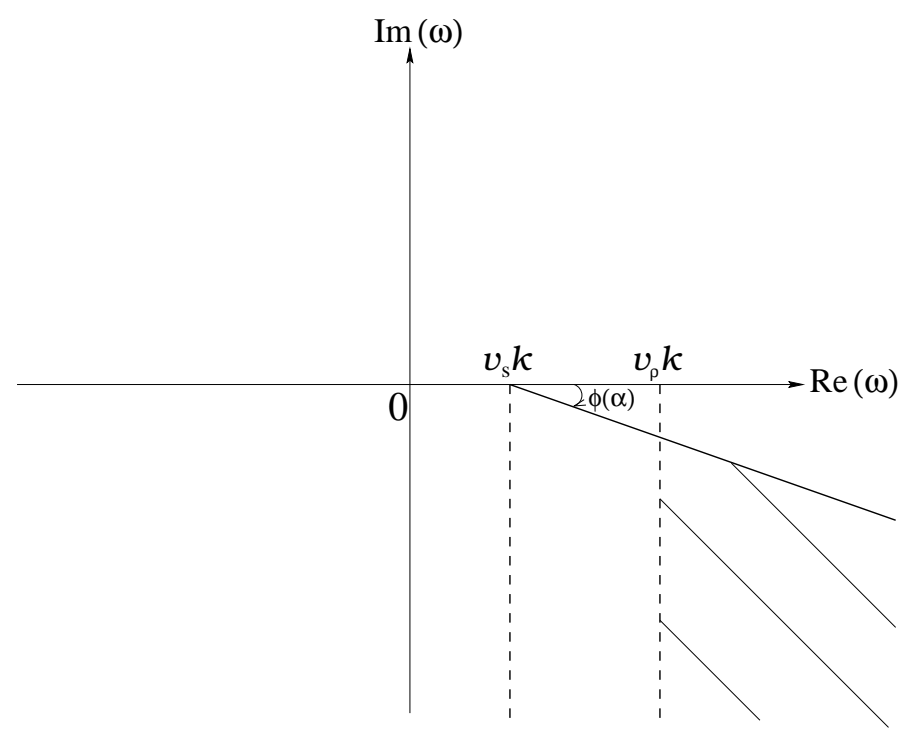

FIG. 11. The restriction of the pole in the shaded region of the complex plane.

where

$$
\begin{array}{r}
\lambda_{c}=\frac{\alpha \pi}{\rho g^{2}(\alpha) h\left(\alpha, \frac{v_{s}}{v_{\rho}}\right)}\left(\frac{\omega_{c}}{\omega_{D}}\right)^{2 \alpha}, \\
h(\alpha, \beta)=\int_{0}^{\frac{\pi}{2}} \frac{\sec ^{2 \alpha} \theta d \theta}{\sqrt{\cos ^{2} \theta+\beta^{2} \sin ^{2} \theta}}, \\
h^{\prime}(\alpha, \beta)=\int_{0}^{\frac{\pi}{2}} \frac{d \theta}{\left[\cos ^{2} \theta+\beta^{2} \sin ^{2} \theta\right]^{\frac{1}{2-2 \alpha}}}
\end{array}
$$

For $d$-wave, the gap is given by $\Delta_{k}=\Delta_{0} \cos \theta_{k}$, where the amplitude $\Delta_{0}$ is

$$
\Delta_{0}=g^{-1}(\alpha) \omega_{D}\left[\frac{2 h\left(\alpha, \frac{v_{s}}{v_{\rho}}\right)}{h^{\prime}\left(\alpha, \frac{v_{s}}{v_{\rho}}\right)(1+\alpha) B\left(\frac{1}{1-\alpha}, \frac{3}{2}-\frac{1}{1-\alpha}\right)}\right]^{\frac{1-\alpha}{2 \alpha}}\left(1-\frac{\lambda_{c}^{\prime}}{\lambda}\right)^{\frac{1-\alpha}{2 \alpha}}
$$

The critical coupling $\lambda_{c}^{\prime}=2 \lambda_{c}$. The equation for the transition temperature, $T_{c}$, is unchanged from the case without spin-charge separation, provided the present definitions of the critical couplings, $\lambda_{c}$ and $\lambda_{c}^{\prime}$ are noted.

We find that the quasiparticles are damped, and the real part of their energy is greater than $\left|v_{\rho} k\right|$. Close to $\omega=0$, the density of states, $N(\omega)$, is smaller than that of the normal state. For $s$-wave pairing, we get

$$
N(0)=\rho\left[1-(1-\alpha) B\left(\frac{2-\alpha}{2-2 \alpha}, \frac{2-3 \alpha}{2-2 \alpha}\right)\left(\frac{g(\alpha) \Delta}{\omega_{c}} \sqrt{\frac{v_{\rho}}{v_{s}}}\right)^{\frac{\alpha}{1-\alpha}}\right]
$$


and

$$
\frac{N(\omega)-N(0)}{\rho} \propto \alpha \frac{|\omega|^{2-2 \alpha} \omega_{c}^{\alpha}}{\Delta^{2}}
$$

For $d$-wave pairing, we get

$$
N(0)=\rho\left[1-\frac{1}{2} B\left(\frac{1}{2-2 \alpha}, \frac{2-3 \alpha}{2-2 \alpha}\right)\left(\frac{g(\alpha) \Delta_{0}}{\omega_{c}} \sqrt{\frac{v_{\rho}}{v_{s}}}\right)^{\frac{\alpha}{1-\alpha}}\right]
$$

and

$$
\frac{N(\omega)-N(0)}{\rho} \propto \frac{|\omega|}{\Delta_{0}}
$$

As before, the linearity of the $d$-wave density of states is unaffected by $\alpha$.

We conclude that the spin-charge separated model behaves qualitatively similarly to the model without spin-charge separation. However, we found one important difference between the two models with respect to the coherence of tunneling between two layers in the normal state. In the spin-charge separated model, the coherence is absent if $\alpha>\frac{1}{4}$, and the tunneling

matrix element $t_{\perp}(k)$ is less than a crtical value. Given the anisotropy of $t_{\perp}(k)$ [9], this can lead to interesting results.

\section{CONCLUSIONS}

In the present section we shall summarize our work and point out the directions in which our theory must be improved and extended. We have shown that the notion of spectral anomaly is a powerful one. It allows us to formulate a framework with which we can understand non-Fermi liquid effects on superconductivity, regardless of the microscopic origin of the spectral anomaly. We believe that any low energy effective field theory of gapless fermions can be characterized by an anomalous spectral function that reflects the critical state of the fermions. The results obtained from the spin-charge separated model are qualitatively similar to those obtained from the model wthout spin-charge separation. However, the single particle tunneling between the layers shows a greater degree of incoherence in the spin-charge separated model. We have also shown that the concept of spectral anomaly 
provides a basis for understanding the features of the interlayer tunneling theory of high temperature superconductors.

Spectral anomaly has two important effects. It makes the the single particle spectrum incoherent while retaining some of the features of a Fermi liquid. The spectrum is gapless and therefore the system responds as a metal: an infinitesimal electric field generates a conducting response. We have also seen that one can still define a surface in the momentum space at which the nature of the excitations changes, as at the Fermi surface of a Fermi liquid. However, the momentum distribution is not discontinuous at this surface but its derivative is singular. Spectral anomaly has also the negative effect that conventional BCS pairing is irrelevant (in the renormalization group sense). We have seen that one needs a coupling larger than a critical coupling to achieve superconductivity. Nonetheless, the interlayer pairtunneling Hamiltonian is relevant (not marginal). This is particularly interesting because many of the features are quite distinctive and reflect the dominating effect of the interlayer tunneling rather than the small residual in-plane attractive mechanism, which can of course have any symmetry. According to the present analysis, the c-axis tunneling density of states is finite at zero frequency, but a well-defined peak still persists at a finite frequency unless the anomaly exponent, $\alpha$, is very large. While such effects are reported, it is not clear if these are experimental artifacts or not. However, if such effects are not artifacts and can be measured precisely, we can infer the value of $\alpha$ from such experiments.

In the present paper we have been unable to discuss the renormalization of the composite operators such as current. Although we have used the Ward identity to indicate how the vertex should scale, it has not been possible to go further. In our opinion, it is not possible to do so without more powerful methods, such as the unitarity expansion formulated many years ago by Gribov, Migdal, and Polyakov [35]. The renormalization of composite operators is important because most experimental probes couple to composite operators. In this respect, it is necessary to go beyond the leading scaling forms, as the dependences on temperature and frequency are often determined by the the presence of dangerously irrelevant operators as argued by Nayak and Wilczek [36]. 
Our justification of the interlayer tunneling Hamiltonian consisted of a two step process. We first showed that the single particle tunneling is incoherent in a non-Fermi liquid for sufficiently large $\alpha$, which need not, however, be much larger than $\frac{1}{2}$ to $\frac{1}{4}$. We then argued that because of incoherence such a term should not be included in the effective low energy theory. Renormalization group arguments, similar to those discussed in our paper show, however, that such a term is relevant as long as $\alpha<1$. Josephson pair tunneling was then argued not to be severely affected by the spectral anomaly and can be cast into an effective low energy Hamiltonian. It can be argued that it should be possible to begin with a single particle tunneling Hamiltonian and arrive at a low energy theory in which single particle tunneling is absent but pair tunneling is present as a result of correlation effects that give rise to spectral anomaly. Our inability to do so is intimately intertwined with the neglect of vertex corrections in the tunneling process as was remarked by Wen [11].

Ultimately, it will be necessary to provide a microscopic derivation of the spectral anomaly. Beginning with the pioneering work of Anderson [2] many authors have investigated the notion of tomographic Luttinger liquid in two dimensions. Similarly, many authors have focused on an underlying gauge theory of strongly correlated electrons that could possibly give rise to non-Fermi liquid behavior. Recently Khlebnikov [12] has approached interlayer pair tunneling from this perspective. Such ideas seem promising to us.

\section{ACKNOWLEDGMENTS}

We thank P. W. Anderson for numerous discussions. In addition, the chapters of his forthcoming book, available to us for more than several years, have been greatly useful. We

also thank E. Abrahams for pointing out an error made by one of us (S. C.) earlier. A part of this work was carried out at the Aspen center for physics. This work was supported by the National Science Foundation, NSF-DMR-92-20416. 


\section{APPENDIX A: SPECTRAL FUNCTION I}

We derive here the spectral function corresponding to one particle green's function of the interlayer tunneling problem discussed in Sec. (IIIA).

$$
A_{i j}(k, \omega)=-\frac{1}{\pi} \operatorname{Sign}(\omega) \operatorname{Im}[G(k, \omega)]_{i j},
$$

where for both the regions $\varepsilon(k)<\omega<0$ and $\omega<\varepsilon(k), \omega<0$,

$$
\operatorname{Im}[G(k, \omega)]_{11}=\frac{\sin \left(\frac{\alpha \pi}{2}\right) D^{-}(k, \omega)^{1-\alpha}\left[t_{\perp}^{2}(k)+D^{-}(k, \omega)^{2(1-\alpha)}\right]}{\left[D^{-}(k, \omega)^{2(1-\alpha)}-\cos (\alpha \pi) t_{\perp}^{2}(k)\right]^{2}+\sin ^{2}(\alpha \pi) t_{\perp}^{4}(k)} .
$$

For the regions $\omega>0, \omega>\varepsilon(k)$ and $0<\omega<\varepsilon(k), \operatorname{Im}[G(k, \omega)]_{11}$ is negative of the expression given in Eq. ( A2). In the more compact form, the spectral function is

$$
A_{11}(k, \omega)=\frac{1}{t_{\perp}(k)} F_{11}\left(\frac{\omega-\varepsilon(k)}{t_{\mathrm{eff}}(k)}\right),
$$

where the dimensionless function $F_{11}(x)$ is given by

$$
F_{11}(x)=\frac{\sin \left(\frac{\alpha \pi}{2}\right)|x|^{1-\alpha}\left[1+|x|^{2(1-\alpha)}\right]}{\pi\left[|x|^{4(1-\alpha)}-2 \cos (\alpha \pi)|x|^{2(1-\alpha)}+1\right]} .
$$

Similarly, the spectral function related to tunneling between the layers is related to the off-diagonal element by

$$
A_{12}(k, \omega)=-\frac{1}{\pi} \operatorname{Sign}(\omega) \operatorname{Im}[G(k, \omega)]_{12},
$$

where, for $\varepsilon(k)<\omega<0$,

$$
\operatorname{Im}[G(k, \omega)]_{12}=\frac{\sin (\alpha \pi) D^{-}(k, \omega)^{2(1-\alpha)} t_{\perp}(k)}{\left[D^{-}(k, \omega)^{2(1-\alpha)}-\cos (\alpha \pi) t_{\perp}^{2}(k)\right]^{2}+\sin ^{2}(\alpha \pi) t_{\perp}^{4}(k)} .
$$

In the region $\omega<0, \omega<\varepsilon(k), \operatorname{Im}[G(k, \omega)]_{12}$ is negative of that given in Eq. (A6). In the region $\omega>0, \omega>\varepsilon(k), \operatorname{Im}[G(k, \omega)]_{12}$ is negative of that given in Eq. ( region $\varepsilon(k)>\omega>0, \operatorname{Im}[G(k, \omega)]_{12}$ is the same as that given in Eq. (A6).

Therefore we have

$$
A_{12}(k, \omega)=\frac{1}{t_{\perp}(k)} F_{12}\left(\frac{\omega-\varepsilon(k)}{t_{\mathrm{eff}}(k)}\right)
$$

where

$$
F_{12}(x)=\frac{\operatorname{Sign}(x) \sin (\alpha \pi)|x|^{2(1-\alpha)}}{\pi\left[\left[|x|^{2(1-\alpha)}-\cos (\alpha \pi)\right]^{2}+1\right]}
$$




\section{APPENDIX B: SPECTRAL FUNCTION II}

It is interesting to calculate the superconducting density of states and the spectral function. First, consider the spectral function. The imaginary part of the matrix element, $\left[G_{s}^{\mathrm{R}}(k, \omega)\right]_{11}$, of the retarded Green's function is related to the spectral function by

$$
A_{s}(k, \omega)=-\frac{1}{\pi} \operatorname{Im}\left[G_{s}^{\mathrm{R}}(k, \omega)\right]_{11} .
$$

In terms of the time-ordered Green's function,

$$
A_{s}(k, \omega)=-\frac{1}{\pi} \operatorname{Sign}(\omega) \operatorname{Im}\left[G_{s}(k, \omega)\right]_{11}
$$

First, consider first $\omega<0$. There are three separate case: $(1) \varepsilon(k)<\omega,(2) \omega<\varepsilon(k)<-\omega$, and $(3)-\omega<\varepsilon(k)$.

1. $\varepsilon(k)<\omega$

In this region, we have $\varepsilon(k)<\omega<0<-\omega$. Therefore, both $\omega-\varepsilon(k)>0$ and $-\omega-\varepsilon(k)>0$. Then,

$$
\operatorname{Im}\left[G_{s}(k, \omega)\right]_{11}=\frac{\sin \left(\frac{\alpha \pi}{2}\right) D^{+}(k, \omega)^{1-\alpha}}{\left[D^{+}(k, \omega) D^{-}(k, \omega)\right]^{(1-\alpha)}+\Delta_{k}^{2}}
$$

2. $\omega<\varepsilon(k)<-\omega$

In this region, there are two possibilities, either $\omega<0<\varepsilon(k)<-\omega$ or $\omega<\varepsilon(k)<$ $0<-\omega$. If we remember that we go counterclockwise around one branch point and clockwise around the other, we get, for both of these cases,

$$
\operatorname{Im}\left[G_{s}(k, \omega)\right]_{11}=\frac{\left.\sin \left(\frac{\alpha \pi}{2}\right) D^{+}(k, \omega)\right)^{1-\alpha}\left[\Delta_{k}^{2}+\left[D^{+}(k, \omega) D^{-}(k, \omega)\right]^{(1-\alpha)}\right]}{\left[\left[D^{+}(k, \omega) D^{-}(k, \omega)\right]^{(1-\alpha)}-\cos (\alpha \pi) \Delta_{k}^{2}\right]^{2}+\sin ^{2}(\alpha \pi) \Delta_{k}^{4}}
$$

3. $-\omega<\varepsilon(k)$

In this region, both $\omega-\varepsilon(k)<0$ and $-\omega-\varepsilon(k)<0$. Therefore

$$
\operatorname{Im}\left[G_{s}(k, \omega)\right]_{11}=\frac{\sin \left(\frac{\alpha \pi}{2}\right) D^{+}(k, \omega)^{1-\alpha}}{\left[D^{+}(k, \omega) D^{-}(k, \omega)\right]^{(1-\alpha)}+\Delta_{k}^{2}}
$$


The case $\omega>0$ can be treated identically and we give the results below, written in a more concise form.

1. $|\varepsilon(k)|>\omega$.

$$
\operatorname{Im}\left[G_{s}(k, \omega)\right]_{11}=-\frac{\sin \left(\frac{\alpha \pi}{2}\right) D^{+}(k, \omega)^{1-\alpha}}{\left[D^{+}(k, \omega) D^{-}(k, \omega)\right]^{(1-\alpha)}+\Delta_{k}^{2}}
$$

2. $|\varepsilon(k)|<\omega$.

$$
\operatorname{Im}\left[G_{s}(k, \omega)\right]_{11}=-\frac{\sin \left(\frac{\alpha \pi}{2}\right) D^{+}(k, \omega)^{1-\alpha}\left[\Delta_{k}^{2}+\left[D^{+}(k, \omega) D^{-}(k, \omega)\right]^{(1-\alpha)}\right]}{\left[\left[D^{+}(k, \omega) D^{-}(k, \omega)\right]^{(1-\alpha)}-\cos (\alpha \pi) \Delta_{k}^{2}\right]^{2}+\sin ^{2}(\alpha \pi) \Delta_{k}^{4}} .
$$

In the scaled form, the spectral weight can be written as

$$
A_{s}(k, \omega)=\frac{1}{\left|\Delta_{k}\right|} F_{s}\left(\frac{\omega}{\left|\Delta_{\mathrm{eff}}(k)\right|}, \frac{\varepsilon(k)}{\left|\Delta_{\mathrm{eff}}(k)\right|}\right),
$$

where

$$
F_{s}(x, y)=\frac{\sin \left(\frac{\alpha \pi}{2}\right)}{\pi}\left[\frac{\theta(|y|-|x|)|x+y|^{1-\alpha}}{\left|x^{2}-y^{2}\right|^{1-\alpha}+1}+\frac{\theta(|x|-|y|)|x+y|^{1-\alpha}\left(1+\left|x^{2}-y^{2}\right|^{1-\alpha}\right)}{\left[\left|x^{2}-y^{2}\right|^{1-\alpha}-\cos (\alpha \pi)\right]^{2}+\sin ^{2}(\alpha \pi)}\right] .
$$

The BCS result, for comparison, is

$$
A_{\mathrm{BCS}}(k, \omega)=u_{k}^{2} \delta\left(\omega-E_{k}\right)+v_{k}^{2} \delta\left(\omega+E_{k}\right),
$$

where $E_{k}=\sqrt{\varepsilon^{2}(k)+\Delta_{k}^{2}}$, and

$$
\begin{aligned}
& u_{k}^{2}=\frac{1}{2}\left(1+\frac{\varepsilon(k)}{E_{k}}\right), \\
& v_{k}^{2}=\frac{1}{2}\left(1-\frac{\varepsilon(k)}{E_{k}}\right) .
\end{aligned}
$$

The superconducting density of states, $N(\omega)$, is

$$
N(\omega)=-\frac{1}{\pi} \operatorname{Sign}(\omega) \sum_{k} \operatorname{Im}\left[G_{s}(k, \omega)\right]_{11}
$$




\section{REFERENCES}

[1] P. W. Anderson, Science 256, 1526 (1992).

[2] P. W. Anderson, Phys. Rev. Lett. 64, 1839 (1990); ibid 65, 2306 (1990).

[3] C. M. Varma, P. B. Littlewood, S. Schmitt-Rink, E. Abrahams, and A. E. Ruckenstein, Phys. Rev. Lett. 63, 1996 (1989).

[4] D. C. Mattis and E. H. Lieb, J. Math. Phys. 6, 304 (1995).

[5] V. J. Emery, in Low-dimensional conductors and superconductors, edited by D. Jérome and L. G. Caron (Plenum, New York, 1987) and references therein.

[6] H. J. Schulz, Les Houches lecture notes, preprint (cond-mat/9503150) and references therein.

[7] F. D. M. Haldane, J. Phys. C14, 2585 (1981).

[8] P. W. Anderson, a forthcoming book, to be published.

[9] S. Chakravarty, A. Sudbø, P. W. Anderson, and S. Strong, Science 261, 337 (1993).

[10] S. Chakravarty and P. W. Anderson, Phys. Rev. Lett. 72, 3859 (1994).

[11] X. G. Wen, Phys. Rev. B 42, 6623 (1990).

[12] S. Khlebnikov, preprint (cond-mat/9508050).

[13] V. Galitski and A. Migdal, Sov. Phys. JETP 7, 96 (1958).

[14] A. L. Fetter and J. D. Walecka, Quantum theory of many- particle systems (McGrawHill, New York, 1971).

[15] C. Nayak and F. Wilczek, Nucl. Phys. B 417, 359 (1994); C. Nayak and F. Wilczek, Nucl. Phys. B 430, 534 (1994); S. Chakrvarty, R. E. Norton, O. F. Syljuåsen, Phys. Rev. Lett. 74, 1423 (1995); M. Onoda, I. Ichinose, T. Matsui, preprint (UT-Komaba $95-1)$. 
[16] V. Meden and K. Schönhammer, Phys. Rev. B 46, 15753 (1992).

[17] J. Voit, Phys. Rev. B 47, 6740 (1993); J. Phys. Condens. Matter 5, 8305 (1993).

[18] G. Benfatto and G. Gallavotti, Renormalization group (Princeton university press, Princeton, 1995).

[19] R. Shankar, Rev. Mod. Phys. 66, 129 (1994).

[20] J. Polchinski, in Recent directions in particle theory, Proc. of 1992 Tasi, eds. J. Harvey and J. Polchinski (World Scientifc, Singapore, 1993).

[21] J. R. Schrieffer, Superconductivity (The Benjamin/Cummings, Reading, 1964).

[22] For another approach, see D. G. Clarke, S. P. Stromg, and P. W. Anderson, Phys. Rev. Lett. 72, 3218 (1994).

[23] V. N. Popov, Functional integrals and collective excitations (Cambridge university press, Cambridge, 1987).

[24] E. Abrahams, private communications.

[25] A. Sudbø, Phys. Rev. Lett. 74, 2575 (1995).

[26] V. N. Muthukumar, D. Sa, and M. Sardar, preprint.

[27] A. Balatsky, Phil. Mag. Lett 68, 251 (1993).

[28] V. Ambegaokar and A. Baratoff, Phys. Rev. Lett. 10, 486 (1963); ibid, 11, 104 (1963), erratum.

[29] L. G. Aslamazov and A. I. Larkin, Pis'ma v Zh. Eksp. Teor. Fiz. 9, 150 (1969) [JETP Lett. 9, 87 (1969)].

[30] A. I. Liechtenstein, O. Gunnarsson, O. K. Andersen, and R. M. Martin, preprint (condmat/9509101). 
[31] W. -C. Wu and A. Griffin, Phys. Rev. Lett. 74, 158 (1994); L. Yin, O. Syljuåsen, and S. Chakravarty, unpublished.

[32] See, for example, J. Yu and A. J. Freeman, J. Phys. Chem. Solids 52, 1351 (1991).

[33] Ch. Renner and Ø. Fischer, Phys. Rev. B 51, 9208 (1995).

[34] Y. Ren and P. W. Anderson, Phys. Rev. B 48, 16662 (1993).

[35] V. N. Gribov and A. A. Migdal, Sov. Phys. JETP 28, 784 (1969); A. A. Migdal, Sov. Phys. JETP 32, 552 (1971); A. M. Polyakov, Sov. Phys. JETP 32, 296 (1971); Sov. Phys. JETP 30, 151 (1970); Sov. Phys. JETP 28, 533 (1969).

[36] C. Nayak and F. Wilczek, preprint (cond-mat/9507040). 